\title{
Propuesta de un protocolo de evaluación de la calidad ecológica de ríos andinos (CERA) y su aplicación a dos cuencas en Ecuador y Perú
}

\author{
Raúl Acosta *, Blanca Ríos ${ }^{1}$, Maria Rieradevall ${ }^{2}$ y Narcís Prat ${ }^{3}$
}

Grupo de Investigación FEM (Freshwater Ecology and Management). Departament d'Ecologia. Universitat de Barcelona. Diagonal 645. 08028. Barcelona

1 brios@ub.edu

2 mrieradevall@ub.edu

3 nprat@ub.edu

* Autor responsable de la correspondencia: racosta@ub.edu

Recibido: 10/6/08

Aceptado: $1 / 10 / 08$

\begin{abstract}
Proposal for an evaluation protocol of the ecological quality of Andean rivers (CERA) and its use in two basins in Ecuador and Peru

A Rapid Protocol is presented for Evaluation of the Ecological Status of Andean Rivers (CERA) localized over 2000 m.a.s.l. from the Northern Andes (Venezuela) through the Altiplano in the Central Andes (Bolivia). This protocol was used in 45 sampling sites in the Guayllabamba River Basin in Ecuador and in 42 sampling sites in the Cañete River Basin in Peru. Previously, and in order to test if the sampling stations may or not be considered reference stations, we constructed a method that assesses 24 basin attributes, hydrology, reach and riverbed and that fluctuates from 24 to 120 points; sites with values higher than 100 were considered as potential reference sites.

Besides the benthic macroinvertebrats' evaluation, the river habitat and riparian vegetation were also evaluated through of the application of the indices ABI (Ríos et al., submitted), IHF (Pardo et al., 2002) and QBR-And, respectively. The convenience of the initial allocation of the reference sites was evaluated as well. These indices have been properly adapted to the conditions and characteristics of the high Andes rivers. The results obtained for both basins were compared and discussed. Through the use of the CERA protocol, the particular perturbation gradients and the natural variability of the reference sites in both countries were recognized.
\end{abstract}

Key words: South America, Ecuador, Peru, High Andes rivers, reference conditions, aquatic macroinvertebrates, Andean Biotic Index (ABI), QBR-And, IHF, ECOSTRIAND.

\section{RESUMEN}

Propuesta de un protocolo de evaluación de la calidad ecológica de ríos andinos (CERA) y su aplicación a dos cuencas en Ecuador y Perú

Se presenta un protocolo rápido de evaluación de la Calidad Ecológica de Ríos Andinos (CERA), situados sobre los 2000 m.s.n.m, desde los Andes del Norte (Venezuela) hasta el Altiplano de los Andes Centrales (Bolivia). Este protocolo ha sido aplicado en 45 estaciones de muestreo en la cuenca del río Guayllabamba en Ecuador y en 42 estaciones de muestreo en la cuenca del río Cañete en Perú. Previamente, para probar si las estaciones de muestreo pueden o no ser estaciones de referencia construimos un método que valora 24 atributos de cuenca, hidrología, tramo y lecho y que fluctúa de 24 a 120 puntos; valores superiores a 100 fueron considerados como sitios potencialmente de referencia.

Además del estudio de los macroinvertebrados bentónicos, se evaluó el hábitat fluvial y la comunidad vegetal de ribera a través de la aplicación de los índices ABI (Ríos et al., sometido), IHF (Pardo et al., 2002) y QBR-And respectivamente; así como la conveniencia de la asignación inicial de las estaciones de referencia. Estos índices han sido adecuadamente adaptados a las condiciones y características propias de los ríos altoandinos. Los resultados obtenidos fueron comparados y discutidos entre ambas cuencas. Mediante la aplicación del protocolo CERA se han reconocido los respectivos gradientes de perturbación y la variabilidad natural de las estaciones de referencia en ambos países.

Palabras clave: Sudamérica, Ecuador, Perú, ríos Altoandinos, condiciones de referencia, macroinvertebrados acuáticos, Índice Biótico Andino (ABI), QBR-And, IHF, ECOSTRIAND. 


\section{INTRODUCCIÓN}

Actualmente a nivel mundial existe un creciente interés en preservar los ecosistemas fluviales. Pese a ello, en Latinoamérica se presenta una constante degradación de estos ecosistemas por el aumento de la explotación del recurso y la contaminación de las aguas (Pringle et al., 2000). Particularmente importantes son los ecosistemas fluviales altoandinos ya que proporcionan el suministro de agua a centros urbanos y rurales y la generación de energía eléctrica, entre otros beneficios directos. Sin embargo, la expansión de la frontera agrícola y el aumento de la población humana han incrementado la presión sobre estos ecosistemas y el impacto sobre la calidad del agua (Jacobsen, 1998); de manera que sólo el $5 \%$ de las aguas residuales de la región reciben algún tipo de tratamiento (UNEP, 2002). Las distintas realidades histórico-sociales y de crecimiento económico, ocasionan situaciones de conflicto al no encontrar un equilibrio entre el desarrollo económico de la región y la conservación del medio ambiente (Parra, 1992). Las escasas políticas de saneamiento y la promulgación de leyes de agua demasiado tolerantes con los niveles permisibles de contaminantes orgánicos e inorgánicos, han tenido poco éxito en detener deterioro ambiental existente y la recuperación de los ecosistemas ya deteriorados. Asimismo, esta problemática ambiental conlleva la consecuente pérdida de una biodiversidad que es además, prácticamente desconocida en muchas zonas tropicales.

Una de las principales deficiencias de los estudios de impacto ambiental realizados en Latinoamérica es que la mayoría de ellos han enfatizado solamente el aspecto fisicoquímico del agua; el cual representa solo una de las varias métricas que se deben utilizar para la determinación de la calidad del agua; por lo que es necesario el uso de herramientas integradoras que resuman el efecto global de los principales componentes que conforman el ecosistema acuático (Chapman, 1996; Boon \& Howell, 1997), tal y como ya ha reconocido la Unión Europea mediante la Directiva Marco del Agua (D.O.C.E, 2000).

Algunos estudios en Colombia, Ecuador, Bolivia, Argentina y Chile han utilizado la comuni- dad bentónica para evaluar el efecto de los vertidos orgánicos de ciudades (Roldán et al., 1973; Ballesteros et al., 1997; Machado et al., 1997; Zúñiga de Cardozo et al., 1997; Jacobsen, 1998; Roldán 1999; Monaghan et al., 2000; Posada et al., 2000; Figueroa et al., 2003). Asimismo, la comunidad de macroinvertebrados ha sido reportada como un buen indicador de los impactos producidos por sólidos en suspensión en los Andes Bolivianos (Fossati et al., 2001). Sin embargo, aunque la actividad minera representa una de las principales fuentes de contaminación para los ríos de Perú, Bolivia y el sur de Ecuador (Pringle et al., 2000) y genera una gran cantidad de estudios de impacto ambiental en dichos países, son escasos aquellos que evalúan de forma eficaz su efecto sobre la comunidad de macroinvertebrados bentónicos y son menos aún, aquellos que finalmente son difundidos en forma de publicación científica, pasando la mayoría de ellos a formar parte de una extensa y restringida literatura gris.

Así pues, la falta de métodos eficaces de diagnóstico rápido del estado ecológico, así como la carencia de una validación de dichos métodos reflejada en un protocolo de evaluación; hacen poco comparables los estudios de calidad ecológica de agua entre ríos o entre países, de modo que no se puede llegar a generalizaciones válidas que puedan ser usadas como herramientas por los organismos públicos y privados gestores de los recursos hídricos en los países de la región.

El objetivo de esta publicación es ofrecer una primera versión de una metodología de muestreo y determinación del estado ecológico de los ríos altoandinos que ha desarrollado el grupo de investigación F.E.M. (Freshwater Ecology and Management) del Departamento de Ecología de la Universidad de Barcelona. Esta metodología está basada en los trabajos realizados por el mismo grupo desde 1994 en ríos mediterráneos: protocolo ECOSTRIMED (http: //www.diba.es/mediambient/ecostrimed.asp, Prat et al., 2000) y protocolo GUADALMED (Jáimez Cuéllar, 2002) los cuales han sido convenientemente adaptados a la realidad andina. Este protocolo constituye un primer esfuerzo en generar una herramienta útil para la gestión de los ríos andinos; su uso y aplicación 
futura permitirán consolidar cada uno de sus apartados para la zona de estudio.

El trabajo fue desarrollado para proporcionar unas sencillas herramientas que incluyen varios índices: biológico de macroinvertebrados de calidad de hábitat fluvial (I.H.F., Pardo et al., 2002) y de estado de la vegetación de ribera (QBR-And, modificado a partir del índice QBR, Munné et al., 1998a; Munné et al., 2003). La integración de estos, permite reconocer la calidad de los ríos (ver también Jáimez-Cuéllar et al., 2002). En los apéndices de esta publicación se proporcionan las hojas de campo de los respectivos índices. Una versión más detallada de la aplicación de los mismos se puede encontrar en http://ecostrimed.net. No obstante, a pesar de la sencillez del método, para el caso del ABI y el QBR-And se requiere algunos conocimientos taxonómicos generales de macroinvertebrados acuáticos andinos a nivel de familia y de los árboles y arbustos de ribera más comunes, especialmente de las especies vegetales introducidas en la región. Para ello, hemos incluido en los anexos una lista de los árboles de ribera más comunes, tanto introducidos como nativos. Las claves taxonómicas y dibujos que facilitan el reconocimiento de los macroinvertebrados a nivel de familia pueden ser consultadas en la página web mencionada anteriormente.

\section{ÁREA DE ESTUDIO}

La Cordillera de los Andes se encuentra ubicada en el extremo occidental de Sudamérica desde el sur de Venezuela hasta la Tierra de Fuego y constituye una de las cadenas montañosas más extensas del planeta. Se pueden diferenciar en ella: Andes del Norte, Centrales y del Sur. Los Andes del Norte se ubican desde el sur de Venezuela hasta la depresión de Huancabamba, en el Norte de Perú (5 $5^{\circ}$ lat. S.), atravesando los territorios de Colombia y Ecuador. Es una de las regiones tectónicas mas activas del continente debido a la convergencia de tres placas: la de Nazca, la Sudamericana y la del Caribe. Particularmente importante es la depresión de Huancabamba, porción de la cordillera con mas baja elevación y que representa una barrera que divide biogeograficamente los Andes en dos porciones, siendo considerada como un foco de endemismos y de cambios entre las formaciones vegetales altoandinas (Myers et al., 2000).

Los Andes centrales se extienden desde los 5 a $33^{\circ}$ lat. S., es el segmento más largo y alto de toda la cordillera, recorriendo Perú, Bolivia, y el norte de Argentina y Chile. Comprende tres sectores: Perú central $\left(5-14^{\circ} \mathrm{S}\right)$, AltiplanoPuna (14-26 $\mathrm{S})$ y Sierras Pampeanas (26-33 $\mathrm{S})$. Finalmente, los Andes del Sur se ubican desde $\operatorname{los} 33^{\circ}$ lat S. hasta el extremo del continente en la Tierra de Fuego (Corvalán, 1990; Gregory-Wodzicki 2000; Argollo, 2006).

La compleja topografía y climatología de la región ha favorecido la presencia de distintas formaciones vegetales que pueden ser encontradas a lo largo del área de estudio de este protocolo, cuyo reconocimiento es de gran importancia, ya que ellas no sólo determinan la estructura de la ribera de los ríos, sino que influyen en la composición trófica de las comunidades de macroinvertebrados acuáticos. Algunas de las formaciones mas conspicuas son los Bosques Secos Interandinos (Aguirre et al., 2006) y los Bosques Montanos Húmedos, considerados como uno de los principales centros de diversidad en el mundo (Churchill et al., 1995; Araujo-Murakami \& Zenteno-Ruiz, 2006; Young, 2006). Sobre los $3800 \mathrm{~m}$ snm la cobertura normalmente disminuye notablemente, generando formaciones vegetales características como son los Páramos (Mena \& Hofstede, 2006) y Punas (García \& Beck, 2006).

Los páramos están situados en los Andes del Norte, sobre la línea de los bosques andinos y llegan hasta debajo las nieves perpetuas; desde la Sierra Nevada de Santa Marta en Colombia y la Cordillera de Mérida en Venezuela, hasta la depresión de Huancabamba en el norte de Perú. Constituyen un componente importante de la diversidad de Venezuela, Colombia, Ecuador y Perú (Mena \& Hofstede, 2006) y se diferencian de la Puna por ser más húmedos y tener una mayor cobertura vegetal no estacional (Sánchez-Vega \& Dillon, 2006). El ecosistema de Puna comprende una extensa región de los Andes Centrales situada entre el norte de Argentina y la parte central del Perú dominada por especies herbáceas, en su mayoría gramíneas y al- 
gunas asociaciones de arbustos y árboles de bajo porte. En el anexo 1 se presentan algunas de las principales comunidades vegetales características de los páramos y punas.

La introducción de la ganadería de ovinos y vacunos, la quema del pajonal para que surjan plantas jóvenes más apetecibles para el ganado exótico; el avance de la frontera agrícola a grandes altitudes; la plantación de especies arbóreas exóticas como Pinus spp. y Eucalyptus spp., y otras actividades como el turismo y la minería, han generado una situación de creciente impacto y amenaza para los ecosistemas de páramos y punas. Este impacto, aparte de los daños sobre la biodiversidad y el ambiente, se manifiesta en un descenso en la calidad de vida tanto de las comunidades campesinas marginadas, que viven directamente del ecosistema, como de los millones de personas que usan el agua (cada vez de menor cantidad y calidad) para riego, agua potable e hidroelectricidad en las tierras bajas.

En los páramos y punas, la intervención humana que se inició en tiempos precolombinos y se intensificó posteriormente, ocasionó un grave deterioro en las comunidades vegetales y en muchas zonas las formaciones vegetales están representadas hoy en día por comunidades secundarias de gramíneas, matorrales y turberas (bofedales). Muchos de los bosques relictos de Polylepis spp. se encuentran limitados a zonas de poca accesibilidad generalmente sobre los 4000 metros (Kessler, 2006). Aunque recientemente se ha mencionado que también existen limitaciones ecofisiológicas ya que esta especie no prospera en zonas inundadas como turberas, propios del altiplano de Perú y Bolivia y prefieren quebradas encajonadas con suelos rocosos y pendientes pronunciadas (Kessler, 2006).

Por lo tanto, la presencia de bosques relictos altoandinos no es imprescindible para definir un estado de conservación óptimo de la ribera en las punas y páramos, ya que este puede conseguirse también con los pajonales y/o matorrales, ya sean naturales o producto de una antigua intervención humana, considerando la baja tasa de germinación, crecimiento lento y supervivencia de los árboles nativos.

\section{METODOLOGÍA}

Dentro del marco del proyecto CERA (Calidad Ecológica de Ríos Andinos), el presente protocolo ha sido desarrollado en base primero a una exhaustiva revisión bibliográfica tanto de la comunidad de macroinvertebrados bentónicos como de las comunidades vegetales presentes sobre los 2000 metros de altitud, comprendiendo los andes del norte y centrales hasta el subdominio del Altiplano-Puna a los $26^{\circ} \mathrm{S}$, especialmente para los territorios de Colombia, Ecuador, Perú y Bolivia. Luego, en segundo lugar, en el marco del proyecto CERA (Calidad Ecológica de Ríos Andinos), se evaluaron de forma preliminar algunas cuencas de Ecuador y Perú, durante las estaciones secas de los años 2003, 2004 y 2006.

La distribución de las estaciones de muestreo y su ubicación georeferenciada en ambos países se muestran en la Tabla 1 y el anexo 2 respectivamente. En Ecuador, se evaluaron 45 puntos de muestreo en un rango altitudinal de 2200 a $3800 \mathrm{~m}$ snm y situados en las cuencas de los ríos San Pedro, Pita y Machángara, en el Valle central de los Andes Ecuatorianos del norte, cerca a la ciudad de Quito. Estos ríos pertenecen a la cuenca del río Guayllabamba, el cual desemboca en el Pacífico a través del río Esmeraldas, que tiene un área aproximada de $21000 \mathrm{~km}^{2}$. La cuenca del río san Pedro nace al Sur Occidente de la hoya de Quito y recibe sus aguas de afluentes que nacen en los Illinizas, Corazón, Atacazo, la Viudita, el Rumiñahui y el Pasochoa. La cuenca del río Pita nace al Sur Oriente de la hoya de Quito y recibe sus aguas de afluentes que nacen en El Rumiñahui, el Cotopaxi, el Pasochoa, el Sincholagua, y el Antisana.

En Perú, se evaluaron 42 estaciones ubicadas en la cuenca del río Cañete entre los 2500 y los $4500 \mathrm{~m}$ snm y ubicadas en las subcuencas de los ríos Miraflores, Alis, Laraos, Yauyos, Huantán, Tupe y Lincha, tributarios de la cuenca alta del río Cañete, así como también algunas estaciones ubicadas en las cabeceras y el curso principal del río Cañete. El río Cañete pertenece a la vertiente del Pacífico, se origina de los glaciares de las cordilleras de Ticlla y Pichahuarco a $4830 \mathrm{~m}$ snm. 
Tabla 1. Distribución de las estaciones de muestreo en Ecuador y Perú. Distribution of the sampling sites in Ecuador and Peru.

\begin{tabular}{llc}
\hline País & Cuencas & N $^{\mathbf{0}}$ Estaciones \\
\hline Ecuador & Guayllabamba: & $\mathbf{4 5}$ \\
& San Pedro & 23 \\
& Pita & 15 \\
& Machángara & 7 \\
Perú & Cañete: & $\mathbf{4 2}$ \\
& Cabeceras & 15 \\
& Alis & 7 \\
& Miraflores & 4 \\
& Huantán & 4 \\
& Yauyos & 2 \\
& Laraos & 4 \\
& Tupe & 2 \\
& Lincha & 4 \\
\hline
\end{tabular}

Tiene una extensión de $215 \mathrm{~km}$ y un área aproximada de $6192 \mathrm{~km}^{2}$, surca las provincias de Yauyos y Cañete (Departamento de Lima).

La aplicación del protocolo CERA consta de las siguientes etapas:

\section{Selección de la estación de muestreo}

La selección de las estaciones de muestreo se realizará en base a una cartografía 1:50000 o inferior. En esta fase previa de gabinete se deben tener en cuenta una serie de recomendaciones como que el número de estaciones de muestreo dependerá de la longitud del ríos objeto de estudio y del detalle que queramos conseguir, lo que puede incluir afluentes o no según el objeto de estudio. La distancia entre estaciones no debería ser superior a 10 kilómetros para una red de vigilancia y localizarse en puntos estratégicos para la calidad del agua, como antes y después de las poblaciones principales. El lugar seleccionado en el gabinete se redefinirá en el campo si es necesario, siendo muy importante para esto la facilidad de acceso que presenten las estaciones previstas.

\section{Evaluación de las condiciones de referencia de los ríos andinos}

Para establecer un protocolo de evaluación de la calidad ecológica de un río es necesario contar con información de estaciones de muestreo de referencia. En ese sentido, la Directiva Marco del Agua, indica que las condiciones de referencia deben asignarse según los diferentes tipos de cuerpos de agua y no necesariamente representan condiciones prístinas y totalmente inalteradas, sino que cierta presión humana puede presentarse mientras no existan efectos ecológicos o estos sean mínimos (Wallin et al., 2003).

Existen muchas metodologías para establecer las condiciones de referencia y todas ellas presentan ventajas e inconvenientes. El primer paso es decidir si se seleccionan las estaciones de referencia por las posibles presiones o impactos que en ellas se producen y que son el origen de la mala calidad ecológica de los ríos o al revés, es decir, estudiar directamente las comunidades de organismos y a partir de ellos establecer las condiciones de referencia. En nuestro caso, se seguirá el primer criterio, es decir, se medirá una serie de características que pueden ser origen de la degradación de las comunidades acuáticas. Si esto se produce o no, se validará con las medidas de estado ecológico que tomamos en cada estación (en nuestro caso los índices IHF, QBR-And y ABI). Por esta razón no se pueden usar de forma directa las medidas de estos índices para fijar las condiciones de referencia.

Para los ríos andinos hemos adoptado una metodología que se deriva de nuestros estudios anteriores (Bonada et al., 2002; Chaves et al., 2006) con una cuantificación de las presiones o impactos de forma similar a como se hace en los Índices de Integridad Biótica. Uno de sus principales aportes, es que se incluye una manera de evaluar la condición de referencia de los diferentes puntos de muestreo incorporando una visión en cuatro niveles jerárquicos: cuenca, hidrología, tramo y lecho del río. Esta es una metodología potencial que debe ser testada en más ríos para ver su posible utilidad. Los respectivos apartados de esta evaluación se incluyen en el anexo 3.

Para valorar los términos "Poco", "Medio" o "Mucho" en cada uno de los apartados se indican algunos valores que de forma aproximada pueden usarse. Para el apartado de cuenca puede usarse la información disponible en formato GIS (o bien el Google-earth, fotos aéreas o recursos similares). Para el apartado hidrología debería poder acce- 
derse a la información disponible de la cuenca o sino se estima de acuerdo a la información que se tiene del punto de muestreo. Los apartados tramo y lecho se pueden evaluar mediante una visita o bien con fotografías del lugar.

En los distintos apartados se ha intentado reflejar la amplia variedad de posibles impactos frecuentes en la región andina, desde las actividades ganaderas, agrícolas y mineras, la introducción de especies arbóreas exóticas, la presencia de presas, canalización del río, derivación de aguas, etc., cada una de ellas evaluadas en las respectivas escalas espaciales. El valor máximo del índice es 120 y el mínimo 24. Para que una estación pueda inicialmente ser considerada como de referencia, debe puntuar más de 100 puntos y obtener como mínimo 20 puntos en cada apartado. Así mismo, existen algunas situaciones que considerando la gravedad de su impacto, su presencia en la estación determina inmediatamente que no pueda ser considerada como de referencia, esto básicamente se debe a la explotación minera, la presencia de grandes presas, trasvases, derivaciones para centrales hidroeléctricas y canalización del río.

\section{Toma de muestras}

\section{Evaluación físicoquímica del agua}

Para que el muestreo sea lo más representativo posible las mediciones y tomas de muestras deben realizarse en puntos que representen los tipos de hábitats presentes en el área de estudio, para ello hay que seleccionar zonas donde el agua esté bien mezclada, evitando rebosaderos de los embalses, confluencias de ríos poco importantes, lugares de pequeños vertidos, etc. ya que sólo tienen efectos muy localizados en la química del agua de ese tramo, y evaluarían incorrectamente el estado del río y las características del agua en el tramo de estudio. Las medidas fisicoquímicas y tomas de muestras de agua deben ser tomadas antes o en su defecto, aguas arriba de la zona donde se realicen los otros muestreos (especialmente el biológico) ya que estos pueden alterar algunas de las características del agua.

Debido que el caudal del río puede condicionar las características fisicoquímicas del agua y el tipo de organismos presentes, es importante medirlo. El caudal se calculará al final de los demás muestreos en las localidades donde sea posible, en general en ríos de hasta $10 \mathrm{~m}$ de ancho, donde el canal sea más estrecho. No es recomendable medir este parámetro en los tramos más bajos de los ríos, puesto que la gran profundidad del cauce dificulta la toma de datos. En estos casos, se pueden tomar como caudales de referencia los que provienen de estaciones de aforamiento cercanas. La metodología para calcular el caudal puede consultarse en http://ecostrimed.net.

La temperatura, el oxígeno disuelto, la conductividad y el $\mathrm{pH}$ cambian de manera significativa en cuestión de minutos y por ello fueron determinados en campo. La conductividad y el $\mathrm{pH}$ fueron medidos en ambos países con una sonda YSI-63, mientras el oxígeno y la temperatura con una oxímetro YSI-550A, el cual incluye compensación por temperatura y altitud. También, en cada estación se tomaron muestras de agua para evaluar nutrientes, que fueron llevadas a laboratorios especializados en universidades locales y analizadas mediante los métodos estandarizados de la American Public Health Association (APHA). Para el caso de ríos altoandinos situados a mas de 3000 metros de altitud, hay que considerar que muchos equipos no están diseñados para trabajar por encima de esta altitud, por lo que pueden generar un error que debe ser consultado con las respectivas casas distribuidoras para intentar estimarlo y dar valores mas reales con el uso de algún tipo de factor de corrección. En nuestro caso, el equipo fue inicialmente calibrado en el aire a nivel del mar $(100 \%$ de saturación) y a $62.5 \%$ a partir de los $3900 \mathrm{~m}$ snm, corrección derivada del ajuste proporcionada por tablas del oxímetro YSI.

En el caso de poder hacer análisis fisicoquímicos más completos, como por ejemplo de nutrientes, estos serían útiles para contrastar los datos biológicos, aunque en este protocolo no son imprescindibles.

\section{Evaluación de la calidad del hábitat fluvial (IHF)}

La calidad del hábitat será evaluada a partir del índice IHF (Pardo et al., 2002) el cual básica- 
mente ha sido aplicado sin mayores cambios para la zona de estudio, debido a que la mayoría de las características físicas del hábitat fluvial evaluadas en sus seis apartados son factibles de ser encontradas y valoradas en los ríos altoandinos (Anexo 4). Sin embargo hay que hacer algunas aclaraciones con respecto a dos de sus apartados. En el apartado 5: "Porcentaje de sombra en el cauce", es importante señalar que generalmente sobre los 4000 metros, mucha de la vegetación de ribera en los ríos altoandinos esta representada únicamente por pajonal de gramíneas o matorrales de bajo porte, por lo que es común que estas áreas estén totalmente expuestas a la radiación solar. Esta misma característica determina también la valoración en el apartado 6, correspondiente a los "Elementos de heterogeneidad" ya que algunos de estos elementos como presencia de hojarasca, troncos, ramas y raíces expuestas sólo se presentan a altitudes por debajo de los 4000 metros donde es mas frecuente encontrar una comunidad vegetal de ribera mas compleja. Por lo tanto, estas limitaciones impuestas por la comunidad de ribera deben ser valoradas en la discusión de resultados. En general se ha establecido que los valores del IHF por debajo de 40 indican serias limitaciones de calidad de hábitat para el desarrollo de una comunidad bentónica diversa, siendo el óptimo superior a 75 (Pardo et al., 2002).

\section{Índice de la calidad de la vegetación de ribera Andina (QBR-And)}

El índice QBR ha sido utilizado eficazmente para evaluar la calidad del bosque de ribera en las cuencas mediterráneas (Suárez-Alonso \& Vidal- Abarca, 2000; Suárez-Alonso et al., 2002). La evaluación de la vegetación de ribera andina, se realizará mediante una observación de como máximo 100 metros lineales del río (aunque puede ser menor en ríos pequeños o en el caso de cambios bruscos en las características del río como por ejemplo un salto de agua). En dicho transecto se aplicará una adaptación del índice de calidad de vegetación de ribera, QBR (Munné et al., 1998b y b; Munné et al., 2003) al que llamaremos QBR-And que en su forma más completa incluye cuatro apartados:
Grado de Cubierta de la Ribera, Estructura de la Cubierta, Calidad de la Cubierta y Grado de Naturalidad del Canal Fluvial (Anexo 5).

En términos generales, considerando las principales formaciones vegetales andinas y sus tipos de riberas podemos definir 3 tipos:

Tipo 1: Ribera de tipo rocoso, que no permite el desarrollo de una comunidad vegetal.

Tipo 2: Ribera típica de páramos y punas, conformada por pajonal de gramíneas, en algunos casos con matorrales bajos, almohadillas y turberas de altura (bofedales).

Tipo 3: Ribera conformada por una comunidad arbórea y/o arbustiva muy diversa. Este tipo de ribera es la más frecuente entre los 2000 y $4000 \mathrm{~m}$ snm y en algunos de los bosques relictos sobre los $4000 \mathrm{~m}$ snm en los páramos y punas.

Para las estaciones con riberas del tipo 1, solo se puede aplicar el último apartado del protocolo (grado de naturalidad del canal fluvial), al no poder exigírsele más a la ribera, ya que presentan fuertes consolidados rocosos que imposibilitan el crecimiento vegetal. Entonces, para este tipo de ríos, si el grado de naturalidad del canal es óptimo, obtendría directamente los 100 puntos.

Las evidentes diferencias entre las comunidades vegetales de los pajonales de páramos y punas (tipo 2) y los bosques (tipo 3) hacen necesaria la separación del QBR en dos entradas diferentes para estos tipos de formaciones vegetales.

Para el caso del pajonal de páramos y punas, en el primer apartado (grado de cubierta de la ribera) se incorpora el efecto de una de las actividades que más modifican la cubierta vegetal de orilla: las quemas de pajonal, práctica usual en muchas comunidades andinas realizadas actualmente con el objetivo de mejorar los pastizales y antiguamente con fines de cacería (Kessler \& Driesch 1993; Kessler, 2006). Las siguientes opciones dentro de este apartado se refieren a los porcentajes de cobertura de la ribera y se suman puntos según exista conectividad con la comunidad vegetal adyacente a la ribera.

En las riberas de páramos y punas, al no poder definirse una estructura vegetal adecuada que reper- 
cuta en la dinámica fluvial, se excluye de evaluación el segundo apartado correspondiente a este aspecto. Así mismo, en el tercer apartado, correspondiente a la calidad de la ribera, aunque no es tan frecuente encontrar especies introducidas como Eucalyptus spp. y Pinus spp., es más común la ocurrencia de especies propias de vegetación secundaria, como consecuencia de la actividad ganadera y/o agrícola. Garcia \& Beck (2006) mencionan que en zonas del altiplano degradado por sobrepastoreo prosperan especies vegetales secundarias como Tetraglochin cristatum, Senecio spinosus, Aciachne pulvinata, A. acicularis, y Astraglaus garbancillo. Considerando esto, se recomienda antes de aplicar el QBR en este apartado documentarse sobre las especies vegetales secundarias en las respectivas zonas donde se vaya a aplicar. El cuarto apartado (grado de naturalidad del canal fluvial) no ha sido modificado para los pajonales de páramos y punas, ya que las situaciones planteadas pueden ocurrir indistintamente en bosques o en páramos y punas.

En las riberas de tipo 3, es decir, compuestas por vegetación arbórea/arbustiva, la principal diferencia del QBR-And con respecto al QBR original se encuentra en el tercer apartado correspondiente a la calidad de la cubierta, ya que en el QBR-And queda simplificado a estimar el porcentaje de la cubierta vegetal representado por especies autóctonas e introducidas, independiente del número de las mismas y del tipo geomorfológico de la estación en estudio. Esta modificación responde a la gran diversidad de especies vegetales arbóreas reportadas en las riberas de los ríos andinos, especialmente entre los 2000 y 3000 m snm, muchas de los cuales, a diferencia de las riberas mediterráneas, no son exclusivas de los ecosistemas fluviales. En el anexo 6 se muestran algunas de las principales especies vegetales arbóreas introducidas y autóctonas en los Andes. Los demás apartados en las riberas tipo 3, correspondientes a grado de cubierta, estructura y grado de naturalidad del río se han mantenido sin variaciones.

Cada apartado puede obtener una puntuación máxima de 25 y el total del QBR-And para una ribera tipo 3 perfectamente conservada sería de 100. Sin embargo para las riberas tipo 1 y 2 al no obtener directamente la puntuación máxima, por falta de evaluación de uno (tipo 2) o tres de sus apartados (tipo 1), los valores de cada apartado tendrán que ser ponderados de modo que indirectamente alcancen el valor máximo y así poder ser comparados con las riberas de tipo 3 .

Los rangos de calidad y conservación de las riberas, propuestos para el índice QBR-And se presentan en la Tabla 2.

\section{Muestreo multihábitat de macroinvertebrados y cálculo del Andean Biotic Index (ABI)}

Con fines de biomonitoreo, es aconsejable muestrear tanto en la estación seca como la de lluvias; Jacobsen (1998) encontró que en lugares limpios de los Andes de Ecuador, una mayor cantidad de taxa fueron colectados en la estación seca, mientras en sitios contaminados la mayor riqueza se presentó en la estación de lluvias. En condiciones prístinas o de poca perturbación la menor riqueza de taxa en la estación de lluvias, esta relacionada con la mayor inestabilidad hidrológica durante la época de lluvias, que genera una alta mortalidad de taxa debida a las fuertes avenidas y la consiguiente aparición de comunidades pioneras, de ciclos de vida corto y altas densidades (Jacobsen \& Encalada, 1998). El muestreo de macroinverte-

Tabla 2. Rangos de calidad de conservación de la vegetación de ribera propuestos para el QBR-And. Conservation quality ranks of the riparian vegetation proposed for the $Q B R$-And.

\begin{tabular}{lcc}
\hline Nivel de calidad & QBR-And & Color representativo \\
\hline Vegetación de ribera sin alteraciones. calidad muy buena. estado natural & $\geq 96$ & Azul \\
Vegetación ligeramente perturbado. calidad buena & $76-95$ & Verde \\
Inicio de alteración importante. calidad intermedia & $51-75$ & Amarillo \\
Alteración fuerte. mala calidad & $26-50$ & Naranja \\
Degradación extrema. calidad pésima & $\leq 25$ & Rojo \\
\hline
\end{tabular}


brados debe ser el primero en realizarse, usando una red de mano de $250 \mu$ y en todos los tipos de hábitats presentes en el río (muestreo multihábitat), que es lo que requiere el ABI. No se debe aplicar métodos que usen datos de un solo tipo de hábitat, por ejemplo solo en zonas reófilas, ya que estos sólo describen una parte de la riqueza de la comunidad bentónica.

Después de haber obtenido la muestra, se puede hacer una primera identificación de los organismos en campo y registrar sus rangos de abundancia en la hoja de campo donde se listan las familias de macroinvertebrados que es posible encontrar en los Andes por encima de los $2000 \mathrm{~m} \mathrm{snm}$. El muestreo se repetirá hasta que no aparezcan nuevas familias de macroinvertebrados en la identificación en campo; las claves taxonómicas y dibujos aunque facilitan el reconocimiento de los macroinvertebrados requieren de un cierto conocimiento previo para su correcta utilización. Para las estaciones de referencia, se recomienda llevar las muestras al laboratorio, previo etiquetado y conservación con formol al 10\% (o alcohol al $90 \%$ ) y hacer una separación e identificación de los individuos, ya que de esta manera se puede evaluar el grado de concordancia entre la identificación en campo y la de laboratorio, así como los correspondientes valores del ABI.

$\mathrm{El}$ inventario taxonómico de familias del ABI y sus respectivos valores de tolerancia/sensibilidad se han derivado de publicaciones científicas internacionales y regionales, y literatura gris (reportes técnicos y tesis universitarias no publicadas). La revisión sobre la cual fue construido este índice, incluye desde descripciones taxonómicas de especies hasta estudios ecológicos y de impacto ambiental (Ríos et al., sometido) y su cálculo es similar al del IBMWP (Alba-Tercedor \& Sánchez Ortega, 1988; Alba-Tercedor et al., 2002), el cual constituye una suma de las puntuaciones de todas las familias presentes en el sitio. Los niveles de tolerancia/sensibilidad de cada familia se han derivado principalmente de presiones provenientes de contaminación orgánica (Anexo 7).

Con un determinado nivel de experiencia en la identificación de las familias en campo se puede llegar a obtener un valor del ABI cercano al real sin tener que procesar la muestra en el la- boratorio, esto es especialmente útil en estaciones de no referencia caracterizadas por un baja riqueza de familias. Asimismo, considerando el escaso conocimiento taxonómico de los macroinvertebrados acuáticos altoandinos es importante mantener colecciones de referencia con el fin de posteriores estudios a nivel de género que profundicen en el grado de tolerancia con un mayor nivel de resolución taxonómica. Para una determinación mas detallada de los organismos se aconseja recurrir a bibliografía especializada para la región neotropical (Roldán, 1988; Fernández \& Domínguez 2001) y también revisar los recursos propuestos en www.ecostrimed.net.

\section{Determinación de la calidad ecológica de los ríos andinos}

El ECOSTRIAND (ECOlogical STatus RIver ANDean) es un índice que pretende valorar de forma global la calidad del ecosistema fluvial, incluyendo la ribera además de la calidad de las aguas y de la comunidad de macroinvertebrados. Pertenece a la familia de los índices de evaluación rápida de la calidad del agua, ya que requiere una infraestructura mínima y un tiempo corto de muestreo.

Su cálculo requiere los siguientes pasos:

1. Valorar el índice de hábitat (IHF). Si es superior a 40 puntos podemos asegurar que los valores del índice no van a ser influenciados por la falta de heterogeneidad del hábitat, la cual puede ser tan limitante como la calidad fisicoquímica del agua.

2. Un índice de calidad biológica del río basado en los macroinvertebrados (A.B.I.).

3. El índice de valoración del estado de conservación del sistema de ribera (índice QBR-And).

\section{RESULTADOS}

Los valores de los diferentes índices aplicados para cada estación en ambos países se muestran en el anexo 2. así como también las puntuaciones de referencia obtenidos de acuerdo al protocolo. 
Acosta et al.

Tabla 3. Principales medidas estadísticas de los índices y características químicas de las estaciones de muestreo en Ecuador. Main statistical parameters of the indices and chemical characteristics of sampling sites in Ecuador.

\begin{tabular}{|c|c|c|c|c|c|c|c|c|c|c|c|c|}
\hline & \multicolumn{12}{|c|}{ Ecuador } \\
\hline & \multicolumn{6}{|c|}{ Estaciones de referencia (27) } & \multicolumn{6}{|c|}{ Estaciones alteradas (18) } \\
\hline & Media & Mediana & D.S (Min-Max) & C.V $(\%)$ & $25 \%$ & $75 \%$ & Media & Mediana & D.S (Min-Max) & C.V (\%) & $25 \%$ & $75 \%$ \\
\hline IHF & 82.74 & 85 & $7.32(66-90)$ & 8.85 & 77 & 89 & 57.27 & 57 & $8.29(37-70)$ & 14.48 & 54 & 63 \\
\hline $\mathrm{ABI}$ & 112.52 & 118 & $20.34(68-140)$ & 18.08 & 96 & 128 & 24.61 & 19 & $17.98(3-68)$ & 73.06 & 15 & 26 \\
\hline \# familias & 22.04 & 22 & $2.14(18-25)$ & 9.71 & 21 & 24 & 7.55 & 7 & $4.27(2-16)$ & 56.56 & 5 & 10 \\
\hline QBR & 69.44 & 80 & $21.55(25-100)$ & 31.03 & 60 & 85 & 19.17 & 20 & $8.95(0-30)$ & 46.69 & 20 & 25 \\
\hline $\mathrm{O}_{2}(\mathrm{mg} / \mathrm{l})$ & 7.09 & 6.73 & $1.28(5.54-11.59)$ & 18.05 & 6.15 & 7.87 & 6.50 & 7.19 & $2.16(0.51-8.58)$ & 33.23 & 6.02 & 7.95 \\
\hline $\mathrm{pH}$ & 7.44 & 7.61 & $1.11(2.43-8.55)$ & 14.92 & 7.28 & 8.03 & 7.72 & 7.87 & $0.56(6.52-8.59)$ & 7.25 & 7.44 & 8.15 \\
\hline \multicolumn{13}{|l|}{ Conductividad } \\
\hline$\left(\mu \mathrm{S} \mathrm{cm}^{-1}\right)$ & 166.13 & 148 & $107.15(29.8-463.56)$ & 64.50 & 88.33 & 210.1 & 512.73 & 533 & $216.51(118.9-797)$ & 42.23 & 286.1 & 695 \\
\hline Nitratos (mg/l) & 0.31 & 0.07 & $0.49(0.02-2.14)$ & 158.06 & 0.04 & 0.3 & 1.13 & 1.11 & $0.58(0.05-2.48)$ & 51.33 & 0.74 & 1.59 \\
\hline
\end{tabular}

La variabilidad de los índices en las estaciones de referencia e impactadas en ambos países se graficaron por medio de diagramas de cajas en STATISTICA (6.0) (StatSoft, 1999). Así mismo, las Tablas 3 y 4 resumen los principales estadísticos descriptivos de variabilidad de los índices y de algunas de las variables fisicoquímicas medidas; también se muestran las medias y medianas de las estaciones de referencia e impactadas de las cuencas de ambos países.

\section{Calificación de las estaciones de referencia}

En Ecuador, 27 (60\%) de las 45 estaciones fueron valoradas como de referencia, mientras 18
(40\%) no pueden ser consideradas como tal, básicamente debido a presencia de basuras, contaminación orgánica evidente y derivación del caudal. Las estaciones que no lograron ser consideradas como de referencia se ubican en el río Machángara, y los tramos bajos del San Pedro y Pita, en donde confluyen los vertidos orgánicos de la ciudad de Quito, la acumulación de basura, el efecto de la ganadería y las derivaciones de las aguas para usos urbanos, ganaderos e industriales, entre otras alteraciones importantes. En Perú, de las 42 estaciones de muestreo, 35 fueron consideradas como de referencia $(83.3 \%)$, las siete restantes $(16.4 \%)$ no fueron incluidas debido a graves alteraciones a nivel de desviación del cau-

Tabla 4. Principales medidas estadísticas de los índices y características químicas de las estaciones de muestreo en Perú. Main statistical parameters of the indices and chemical characteristics of sampling sites in Peru.

\begin{tabular}{|c|c|c|c|c|c|c|c|c|c|c|c|c|}
\hline & \multicolumn{12}{|c|}{ Perú } \\
\hline & \multicolumn{6}{|c|}{ Estaciones de referencia (35) } & \multicolumn{6}{|c|}{ Estaciones alteradas (7) } \\
\hline & Media & Mediana & D.S (Min-Max) & C.V $(\%)$ & $25 \%$ & $75 \%$ & Media & Mediana & D.S (Min-Max) & C.V (\%) & $25 \%$ & $75 \%$ \\
\hline IHF & 48.97 & 46 & $15.34(26-87)$ & 31.33 & 38 & 52 & 50.57 & 49 & $9.68(38-67)$ & 19.14 & 42 & 58 \\
\hline $\mathrm{ABI}$ & 85.56 & 80 & $20.20(58-139)$ & 23.61 & 72 & 92 & 38.71 & 45 & $29.63(0-78)$ & 76.54 & 0 & 61 \\
\hline \# familias & 17.26 & 17 & $3.66(11-27)$ & 21.21 & 15 & 19 & 8.43 & 10 & $7(0-14)$ & 83.04 & 0 & 13 \\
\hline QBR & 77.76 & 85 & $15.63(40-100)$ & 20.10 & 65 & 86.45 & 26.43 & 15 & $21.9(0-60)$ & 82.86 & 10 & 45 \\
\hline $\mathrm{O}_{2}(\mathrm{mg} / \mathrm{l})$ & 7.33 & 7.3 & $0.66(6.2-9.6)$ & 9.00 & 7 & 7.5 & 6.90 & 7.16 & $0.86(5.71-7.96)$ & 12.46 & 5.84 & 7.59 \\
\hline $\mathrm{pH}$ & 7.73 & 8 & $0.52(6.7-8.7)$ & 6.73 & 7.2 & 8.12 & 7.67 & 7.6 & $0.51(7-8.3)$ & 6.65 & 7.1 & 8.2 \\
\hline \multicolumn{13}{|l|}{ Conductividad } \\
\hline$\left(\mu \mathrm{S} \mathrm{cm}{ }^{-1}\right)$ & 362.00 & 290 & $255.13(50-1250)$ & 70.48 & 150 & 500 & 254.47 & 249.5 & $131.65(87.5-470)$ & 51.73 & 126.4 & 353.1 \\
\hline Nitratos (mg/l) & 0.46 & 0.62 & $0.48(0-1.86)$ & 104.35 & 0 & 0.62 & 0.29 & 0.29 & $0.24(0-0.63)$ & 82.76 & 0 & 0.47 \\
\hline
\end{tabular}


dal, presencia de basuras (río Yauyos) o actividad minera intensa (río Tomás).

\section{IHF}

En todos los casos el IHF presenta un valor promedio superior a 40, lo que indicaría que el hábitat fluvial es apropiado para albergar una comunidad de macroinvertebrados diversa, presentándose el valor promedio mas bajo (48.97) en las estaciones de referencia de Perú y el mas alto en las estaciones de referencia de Ecuador (82.74), siendo estas las que presentan el menor coeficiente de variabilidad, (C.V) (8.85\%). Así mismo, las estaciones de referencia de Perú, son las que reportan también el mayor C.V (31\%), con un valor mínimo de 26 , lo cual ya esta indicando problemas de falta de hábitat adecuado en algunos de los ríos, aún habiendo sido considerados inicialmente de referencia por falta de presiones.

Al analizar el efecto disgregado en cada una de las subcuencas se observa que esta baja heterogeneidad reflejada en el IHF ocurre principalmente en las estaciones ubicadas en las cabeceras del río Cañete, es decir en el ecosistema de $\mathrm{Pu}-$ na, sobre los 4000 m snm (Fig. 1a). Aún las estaciones consideradas como alteradas, ubicadas en las cuencas del San Pedro, Pita y Machángara en Ecuador y Alis y Yauyos en Perú, no llegan a tener valores tan bajos en el IHF como los encontrados en las estaciones de Puna del río Cañete (Fig. 1b). Los valores mas elevados del IHF se presentan en las estaciones de referencia de las subcuencas de los ríos San Pedro y Pita en Ecuador y Lincha en Perú (Fig. 1a).

\section{QBR-And}

Los principales estadísticos del QBR-And se muestran en las Tablas 3 y 4, y en las figuras 1c y 1d. El valor promedio mas bajo se presenta en las estaciones consideradas como alteradas en Ecuador (19.17) y el más alto en las estaciones de referencia de Perú (77.76). Por otro lado, la mayor variabilidad se presenta en las estaciones impactadas de Perú $(82.86 \%$ C.V), con valores extremos de 0-60 (Fig. 1d). Las riberas de las estaciones de referencia en Perú, obtuvieron una va- riabilidad del $20.1 \%$ C.V, y estuvieron especialmente bien conservadas en las cabeceras del río Miraflores, Alis, y Cañete, usando los protocolos adecuados para cada tipo de río. Por otra parte, las estaciones de referencia de Ecuador presentaron una variabilidad de $31,03 \% \mathrm{C}$.V con valores extremos de 25 a 100, registrándose los valores más altos en las cabeceras del río San Pedro y el más bajo en el río Pita.

\section{Macroinvertebrados y ABI}

El promedio del número de familias de macroinvertebrados en las estaciones alteradas en ambos países fue 8 , siendo las mas frecuentes, Baetidae, Elmidae, Chironomidae y Oligochaeta. Sin embargo, en las estaciones ubicadas río abajo del poblado de Tomas, en la cuenca del río Alis, (Perú), el efecto de la contaminación minera ha sido tan fuerte que inclusive no se colectaron taxas considerados resistentes como Chironomidae y Oligochaeta. Los mayores valores promedio de número de familias se hallaron en las estaciones de referencia en Ecuador, con 22 familias, frente a las 17 de Perú. El mayor coeficiente de variabilidad de las estaciones de referencia de Perú (21.21\%), casi el doble del presentado en Ecuador (11.26\%) nos indica una mayor variabilidad en las referencias de Perú. Sin embargo, los valores máximos del número de familias son similares tanto en Ecuador como en Perú (29 y 27, respectivamente).

Los mayores valores promedio del ABI, se presentaron en las estaciones de referencia en Ecuador (114) frente a las de Perú (85.56) (Figura 1e), con C.V menores de $25 \%$ para ambos países. Por el contrario, en las estaciones alteradas (Figura 1f), los C.V fueron en ambos países superiores al $70 \%$, lo que refleja el amplio rango de los datos, presentándose en Perú algunas estaciones con valores de 0 como en el río Tomas (Alis) hasta 78 en el río Laraos, reflejando distintas presiones de diferentes intensidad: minera y agrícola respectivamente. De la igual manera, se encontraron amplios rangos de variación del ABI en las estaciones alteradas de Ecuador variando de 3 en el río San Pedro y 70 en el Machángara.

La asignación de las distintas clases de cali- 

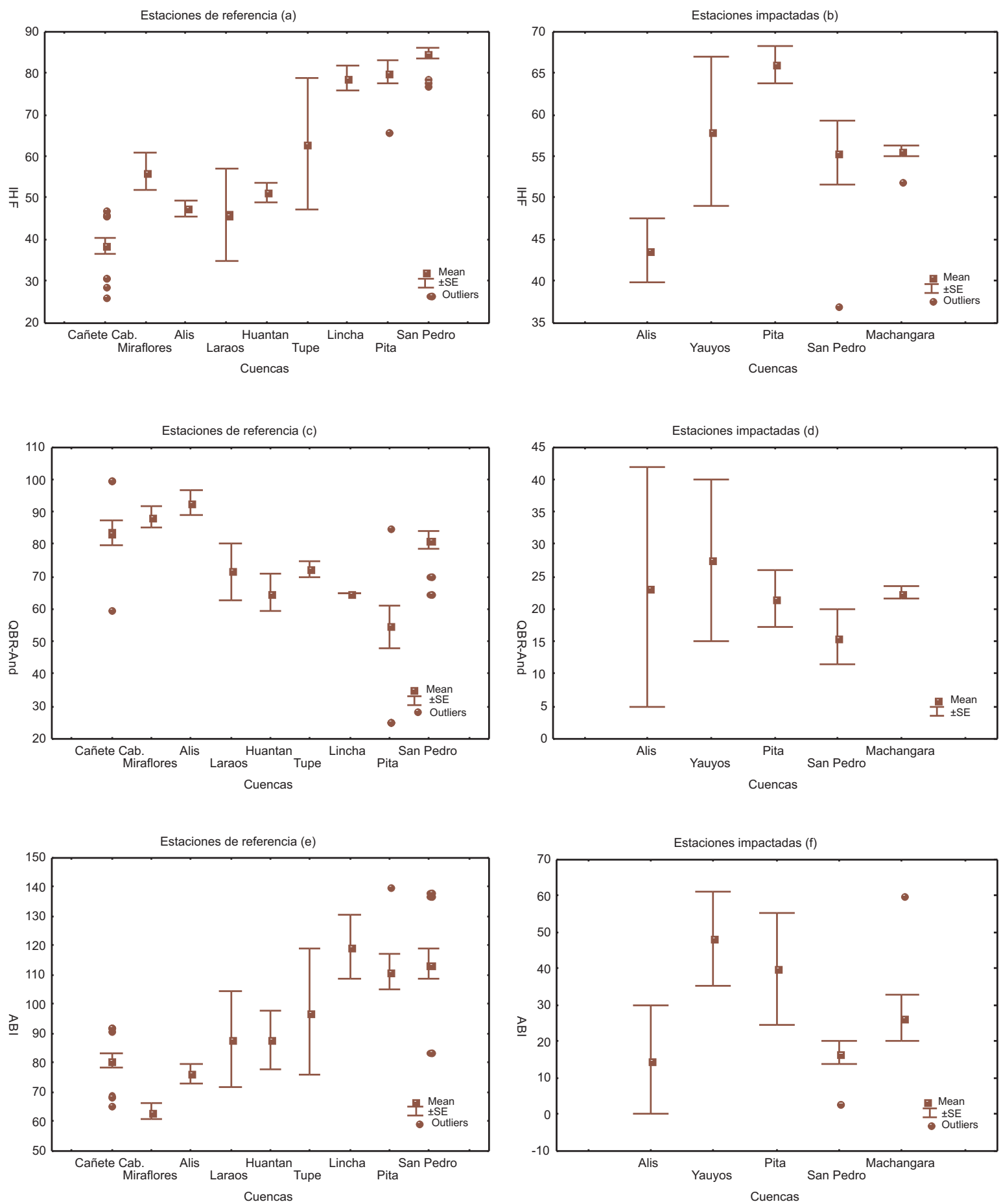

Figura 1. Diagrama de cajas para los índices $\operatorname{IHF}(\mathrm{a}, \mathrm{b}), \mathrm{QBR}-\mathrm{And}$ (c, d) y ABI (e, f) según cuencas en las estaciones de referencia e impactadas. Box Plots for the IHF $(a, b), Q B R$-And $(c, d)$ and $A B I(e, f)$ indices according to basins in the reference and impacted sites.

dad ecológica a partir del ABI, fue diferente en ambos países considerando que las comunidades de referencia eran menos diversas en Perú de forma natural, sin considerar perturbación alguna. 
Tabla 5. Clases de Estado Ecológico según ABI en Ecuador y Perú. Ecological Status classes according to ABI in Ecuador and Peru.

\begin{tabular}{lcc}
\hline & Ecuador & Perú \\
\hline Muy Bueno & $>96$ & $>74$ \\
Bueno & $59-96$ & $45-74$ \\
Moderado & $35-58$ & $27-44$ \\
Malo & $14-34$ & $11-26$ \\
Pésimo & $<14$ & $<11$ \\
\hline
\end{tabular}

En ambos casos, se consideró el valor del percentil 25 de las estaciones de referencia como una ligera desviación de las mismas y por lo tanto una buena aproximación al límite entre las categorías "Muy bueno" y "Bueno". A partir de ello, siguiendo la metodología de la Directiva Marco del Agua (D.O.C.E, 2000; Alba-Tercedor et al. 2002) y considerando que el ABI es una adaptación del BMWP, y este tiene un comportamiento exponencial en relación a los gradientes de alteraciones (Munne \& Prat, 2009, en prensa), los cortes correspondientes a las siguientes categorías de calidad de agua se realizaron también de forma también proporcional al tipo de relación exponencial, definiéndose dichos límites con el 61, 36 y $15 \%$ del percentil 25 de las estaciones de referencia (Tabla 5).

En la Tabla 6 se presenta la valoración del estado de referencia de las estaciones muestreadas en ambos países, mediante el protocolo y el límite superior que representa el estado óptimo de los índices QBR-And, IHF y ABI. Las 18 estaciones designadas a priori como alteradas en Ecuador, coincidieron con valores por debajo del óptimo tanto del ABI como del QBR-And. Mientras en Perú, fueron seis de las siete estaciones consideradas como perturbadas, las que mostraron la misma tendencia y que además coincidieron con limitaciones de hábitat reflejadas en valores del IHF por debajo del óptimo.
En cuanto a las estaciones designadas como de referencia, aunque varias de ellas no alcanzaron el óptimo de estado de conservación de la ribera tanto en Ecuador (13 de 27) como en Perú (14 de 35); un elevado valor del ABI incrementó la calidad ecológica final de algunas de estas estaciones, resultando finalmente que solo tres estaciones para el caso de Ecuador y cuatro para Perú no alcanzaron los limites de óptimos para el ABI y el QBRAnd simultáneamente. Estos puntos de muestreo estuvieron situados, dos en la subcuenca del Pita y una en la del San Pedro, para el caso de Ecuador y en los cursos de los ríos Cañete, Laraos y Huantán en Perú, todos además con limitaciones de hábitat. En general los resultados confirman la eficiente aproximación del protocolo de Estaciones de Referencia en designar posibles estaciones alteradas y de referencia.

Con el índice biológico y de ribera calculados, la calidad ecológica de los ríos (ECOSTRIAND) se obtiene utilizando la Tabla 7, la entrada por la calidad de ribera es la misma para ambas cuencas, pero la entrada por el ABI se separa, ya que como se mencionó anteriormente, las cuencas estudiadas tienen diferentes potenciales de riqueza de familias de macroinvertebrados en condiciones de referencia. El límite inferior del ECOSTRIAND en la columna correspondiente al ABI, se hizo corresponder con el extremo inferior del rango de la calidad ecológica "Moderado" del ABI específica para ambos países.

De esta manera, el valor más importante es el del índice biológico; no es posible considerar que el río tiene un buen estado ecológico si el índice biológico es bajo, a pesar de que la ribera tenga un buen estado; pero el papel de la ribera también es importante, puesto que cuando está degradada, aunque las aguas estén muy limpias, el estado ecológico es regular. La nomenclatura de las

Tabla 6. Comparación entre la propuesta de las estaciones de referencia y los índices QBR-And. IHF y ABI en Ecuador y Perú. Comparison between the proposed reference sites and the indices QBR-And. IHF and ABI in Ecuador and Peru.

\begin{tabular}{lccccc}
\hline Ecuador & $\mathrm{n}$ & QBR-And $<75$ & $\mathrm{IHF}<60$ & ABI $<96$ & ABI $<96$ y QBR-And $<75$ \\
\hline Referencia & 27 & 13 & 0 & 5 & 3 \\
Alteradas & 18 & 18 & 11 & 18 & 18 \\
Perú & $\mathrm{n}$ & $\mathrm{QBR}<75$ & $\mathrm{IHF}<60$ & $\mathrm{ABI}<74$ & ABI $<74$ y QBR-And $<75$ \\
Referencia & 35 & 14 & 28 & 10 & 4 \\
Alteradas & 7 & 7 & 6 & 6 & 6 \\
\hline
\end{tabular}


Tabla 7. Propuesta de establecimiento del Estado Ecológico de las estaciones de muestreo en función de los resultados del ABI y QBR-And (Índice ECOSTRIAND). Proposal for the establishment of the Ecological Status of the sampling sites according to the results of the ABI and QBR-And (ECOSTRIAND Index)

\begin{tabular}{cclll}
\hline ABI $^{1}$ & \multirow{2}{*}{$\mathbf{A B I}^{2}$} & & QBR-And & \\
& & $>75$ & $45-75$ & $<5$ \\
\hline 596 & $>74$ & MUY BUENO & BUENO & REGULAR \\
$59-96$ & $45-74$ & BUENO & REGULAR & MALO \\
$35-58$ & $27-44$ & REGULAR & MALO & PÉSIMO \\
$<35$ & $<27$ & MALO & PÉSIMO & PÉSIMO \\
\hline
\end{tabular}

$\mathbf{A B I}^{1}$ = Estaciones de la cuenca del río Guayllabamba (Ecuador)

$\mathbf{A B I ^ { 2 }}=$ Estaciones de la cuenca del río Cañete (Perú)

cinco clases de estado ecológico que se exponen aquí, derivan de la Directiva Marco de la Unión Europea, las cuales deben seguir siendo validadas posteriormente en los diferentes ecosistemas fluviales andinos del área de estudio. Finalmente, comparando el ECOSTRIAND con las valoraciones del protocolo de Estados de Referencia, sólo una estación ubicada en la subcuenca del río Pita en Ecuador (1.4PI) y otra en la subcuenca del río Huantán (CA-29) en Perú, fueron calificadas originalmente como de referencia y finalmente resultaron como "malas" según el ECOSTRIAND y fue debido principalmente al gran deterioro de la vegetación de sus riberas.
Se realizó un análisis MDS (Multidimensional Scaling) en PRIMER 6.0 (Clarke \& Warwick, 1994) con todas las estaciones de muestreo de Ecuador y Perú (Fig. 2). Las estaciones de referencia y de las cabeceras del río Cañete y de sus subcuencas tributarias del curso alto (Miraflores, Alis, Laraos) se ubican agrupadas en la parte inferior central del diagrama (círculos), las estaciones de referencia de Ecuador se ubican en la parte superior derecha del diagrama (cruces), mezcladas con algunas estaciones de los tributarios de la cuenca media del río Cañete(Lincha, Tupe y algunas de Huantán y Laraos) que por tener una mayor riqueza de familias de macroinvertebrados

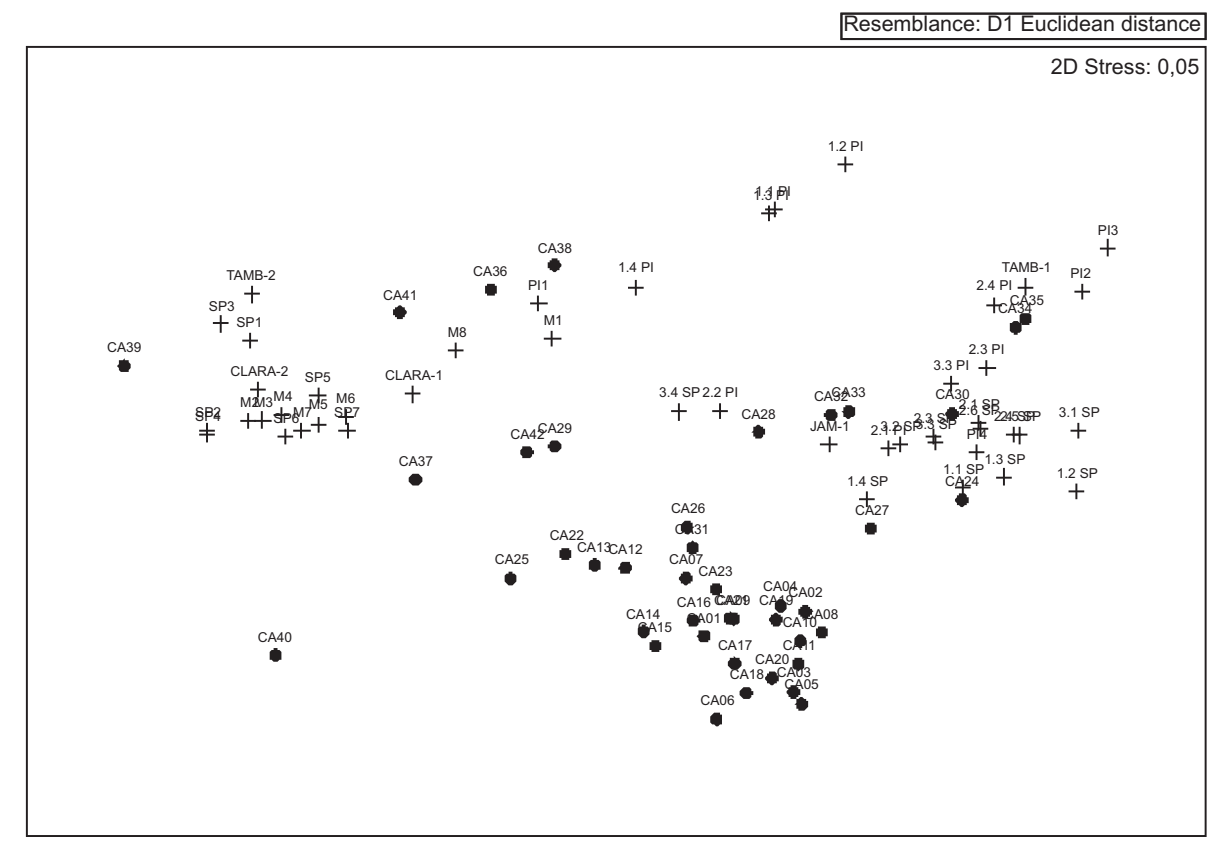

Figura 2. Distribución espacial de las estaciones de muestreo en las cuencas estudiadas, según MDS. Spatial distribution of the sampling stations in the studied basins according to MDS. 
con puntuaciones altas, se acercan mas a las estaciones de referencia de Ecuador. En la zona central superior se ubican las estaciones alteradas de nivel de calidad regular tanto de Ecuador como de Perú. En la parte superior izquierda se ubican muy juntas las estaciones de calidad mala a pésima de Ecuador y finalmente las dos estaciones fuertemente impactadas en Tomas, CA-39 y CA-40 (subcuenca del río Alis, Cañete), con los peores valores de ABI (0) se ubican en la periferia del espacio multidimensional.

\section{DISCUSION}

Las formaciones vegetales dominantes en las punas de los Andes, permiten estudiar un caso evidente en el cual la estructura de la vegetación de ribera no condiciona la estructura de la comunidad de macroinvertebrados. Los bajos valores del IHF presentados en las estaciones de puna de las cabeceras del río Cañete, reflejan en algunas estaciones la baja heterogeneidad del hábitat fluvial y la escasa incorporación de elementos alóctonos provenientes de las riberas del pajonal de gramíneas y pequeños arbustos de la puna. Esta misma composición vegetal de ribera determina que el porcentaje de sombra en estos ríos sea nulo y se encuentren completamente expuestos a la radiación solar, por lo que se esperaría una elevada producción primaria en estos ríos de cabecera, lo cual representaría variaciones a modelos clásicos de funcionamiento y estructura de ríos de cabecera, como el "Río Continuo" (Vannote et al., 1980; Allan, 1995)

La importancia de la heterogeneidad del hábitat fluvial en la ecología de ríos ha sido puesta de manifiesto en muchas ocasiones, tanto en climas templados (Pringle et al., 1988, Palmer \& Poff, 1997; Flory \& Milner, 1999; Beisel et al., 2000; Taniguchi \& Tokeshi, 2004) como en tropicales (Rincón, 1996; Ramirez et al., 1998). En las punas la baja heterogeneidad se corresponde con una escasa oferta de nichos ecológicos, que a su vez condiciona una escasa diversidad de macroinvertebrados bentónicos de forma natural, como lo demuestran los bajos valores del número de familias y del ABI.
Cuando las formaciones vegetales cambian con el descenso de la altitud y dan paso a bosques montanos altos húmedos (Ecuador) o secos (Perú), entre los 2000 y los $3000 \mathrm{~m}$ snm, los valores del IHF se elevan y las comunidades bentónicas se hacen más complejas y diversas. La menor riqueza de familias con el incremento de la altitud en páramos y punas debe considerar también factores históricos, especialmente en algunos taxa como Heteroptera, Coleoptera y Odonata. Jacobsen et al. (1997) señalaron el efecto de las glaciaciones en los Andes como un factor que produjo cambios muy fuertes en las comunidades bentónicos y sugiere que la comunidad presente actualmente es relativamente joven y se encuentra en un proceso de recolonización desde los cursos mas bajos hacia los mas altos, limitados en parte no sólo por las barreras físicas sino también por restricciones térmicas, por lo que actualmente existen muchos nichos ecológicos vacíos dentro del hábitat fluvial altoandino (Jacobsen et al., 2003). Si se incorpora además el efecto de la escasa heterogeneidad del medio, es probable que en su conjunto ambos factores limiten fuertemente la distribución y riqueza de la fauna bentónica altoandina.

Recientemente, otro factor ha sido puesto de manifiesto en cuanto a la baja riqueza de familias presentes en hábitats altoandinos. Jacobsen \& Marin (2007) han reportado una riqueza de familias extremadamente baja en los ecosistemas de la puna del altiplano de Bolivia en comparación a los páramos ecuatorianos y lo han atribuido no sólo a los factores anteriormente mencionados, sino también a las severas fluctuaciones diarias de temperatura y oxígeno disuelto, que implican fuertes presiones fisiológicas limitando una elevada riqueza de familias y especies.

$\mathrm{Al}$ incorporar el efecto de las distintas presiones antrópicas, independientes del tipo de ribera y del hábitat fluvial, la riqueza de familias y especialmente el valor del ABI decae notablemente, hasta llegar a graves extremos de perturbación como son los casos del río Tomas y Yauyos en Perú y San Pedro y Pita en Ecuador. En muchos de estos casos incluso con hábitats potencialmente apropiados (IHF > 40), se presentan valores extremadamente bajos del ABI como en las estaciones bajas del río San Pedro en Ecuador. 
Las estaciones ubicadas en la puna de Perú, donde dominan los pajonales de gramíneas, pueden presentar un QBR-And alto comparable a una ribera forestada de bajas altitudes (entre los 2000 a $3000 \mathrm{~m} \mathrm{snm}$, por ejemplo), debido a que el índice se ha equiparado al máximo potencial que estas riberas pueden mantener considerando las limitaciones altitudinales. En otras áreas como la cabecera del río Miraflores en Perú, aun estando sobre los $4000 \mathrm{~m} \mathrm{snm}$, las características topográficas y la escasa intervención humana, las hacen apropiadas para el desarrollo de bosques de Polylepis spp., por lo tanto estas estaciones se valoran tal como se hubiera hecho en una ribera de menor altitud. Las principales alteraciones de las riberas de bosque están representadas por la presencia de especies introducidas (Eucalyptus spp., Pinus spp.), la disminución de la cobertura vegetal por deforestación, la canalización del río, presencia de estructuras rígidas transversales en el lecho como presas y la presencia de basura. La introducción de Eucalyptus es especialmente importante en las estaciones de la subcuenca de los ríos Huantán, Laraos y Lincha en Perú y en Ecuador está generalizado en prácticamente toda la cuenca del San Pedro y Pita. La presencia de basuras es característica de las estaciones de la cuenca baja del San Pedro, Pita y Machángara en Ecuador y del Yauyos en Perú. La canalización total del río y la presencia de estructuras transversales como puentes se observó en la estación ubicado río abajo del poblado de Tomás en la cuenca del río Alis en Perú.

Aunque las tolerancias del ABI han sido asignadas principalmente en base a presiones de tipo orgánica sobre las comunidades bentónicas, en casos extremos de contaminación minera, como los del río Tomas (Alis), el índice funciona de igual ma nera. Sin embargo hay que tener precaución con niveles intermedios de contaminación minera, cuyos efectos subletales y los umbrales de tolerancia de las familias no han sido aun especificados para los ríos altoandinos. Se sabe por ejemplo que grupos ampliamente conocidos por su sensibilidad a la contaminación orgánica, como los Plecoptera, y Trichoptera pueden sin embargo ser tolerantes a pH ácidos (Winterbourn \& McDiffett, 1996) frecuentes en situaciones de contaminación minera.
Por otro lado, también hay que tener precaución con los típicos efectos de la contaminación orgánica en las zonas altoandinas, especialmente la concentración de oxígeno disuelto, si bien en las estaciones estudiadas no son tan bajas (exceptuando casos extremos como el río Machángara en Ecuador), hay que tener en cuenta que en ríos por encima de los $3000 \mathrm{~m}$ snm los porcentajes de saturación de oxígeno están influenciados por las baja presión parcial del oxígeno en comparación al nivel del mar, lo cual hace que las concentraciones y porcentajes de saturación obtenidos en campo no reflejen las limitaciones reales de las comunidades bentónicas (Jacobsen, 1998). Por lo que estos ríos son más sensibles a contaminaciones de tipo orgánica que ríos de zonas bajas.

En cuanto a la valoración global, podemos mencionar que el ECOSTRIAND acertó mayoritariamente con la valoración inicial de las estaciones de referencia, ya que en todo el estudio, solo dos estaciones, una en cada país, que inicialmente fueron denominadas como de referencia finalmente obtuvieron una calificación de "malas" y tales casos se debieron principalmente a la degradación de la ribera y a limitaciones del hábitat, como la estación CA-29 sobre la subcuenca del río Huantán, en Perú, que al ser un río grande y de elevado caudal, el hábitat es poco heterogéneo, además de las limitaciones del muestreo biológico que ello implica. El análisis MDS agrupó consistentemente las estaciones de muestreo de ambos países según el grado de perturbación y la similitud de sus características generales resumidas en el ABI, IHF y QBR-And. Además, es importante mencionar las similitudes entre las estaciones referencia de Ecuador y las de la cuenca media del río Cañete (Lincha, Tupe, Laraos), las cuales al estar a menor altitud y presentar una mayor heterogeneidad del hábitat fluvial y una vegetación de ribera mas desarrollada y compleja; originan mayores riquezas de macroinvertebrados, muchos de ellos sensibles, lo cual incrementa el ABI, haciéndolas mas semejantes a las estaciones de referencia de Ecuador que a las cabeceras del Cañete.

En esta versión de valoración rápida y simplificada, el índice no considera ni el estado de las comunidades ictícolas, ni los valores de dife- 
rentes parámetros fisicoquímicos, ni la comunidad de vertebrados o diatomeas. Muchos de estos parámetros quedan reflejados, total o parcialmente, en los valores del índice biótico (parámetros fisicoquímicos, peces) o el QBR-And (aves). Las especies alóctonas (como algunos peces -trucha, carpas o tilapias o el cangrejo americano, Procambarus clarkii) deberían ser tenidos en cuenta para la valoración final del estado ecológico y, si están presentes en zonas de un índice ECOSTRIAND alto, éste debería tomarse con precaución. En muchos ríos andinos, el estado de las poblaciones ícticas están compuestas casi exclusivamente de truchas de repoblación y las repercusiones de las especies alóctonas sobre los ecosistemas, son todavía desconocidas. Por ejemplo en el ámbito de un parque natural podríamos pensar que el objetivo final debería ser eliminar las truchas u otras especies introducidas, mientras que en otras áreas, dado el valor económico que puedan tener las truchas para la población local, este objetivo podría no ser el mas importante.

Finalmente, hay que hacer notar que lo presentado en esta publicación es una primera aproximación de un protocolo que se tiene que validar y/o modificar a medida que nuevas cuencas puedan ser tratadas; incorporando otros factores de variabilidad de distinto origen como por ejemplo, tipos de formaciones vegetales, variaciones latitudinales, vertientes y tipos de presiones antrópicas. Las evaluaciones de ríos provenientes de distintas formaciones vegetales son de gran importancia ya que estas comunidades intrínsecamente incorporan en su composición y estructura florística el efecto de otros tipos de variables abióticas como temperatura, precipitación y altitud. El objetivo último será obtener un pro- tocolo de rápido uso y del cual se pueda obtener información confiable de la calidad ecológica de los ríos altoandinos, y que a la vez discierna el efecto de los principales factores de variabilidad natural y antrópica.

\section{AGRADECIMIENTOS}

Los autores agradecerán todas las observaciones sobre el uso de este protocolo que les puedan llegar, tanto directamente como a través de la página web ECOSTRIMED (http://www.ub.edu/ecostrimed) donde el grupo F.E.M. mantiene su base de datos y su metodología a disposición pública.

Para la elaboración de este protocolo se ha contado con la inestimable ayuda de diferentes colaboradores entre los que destacamos:

Andrea Encalada, Carolina Arroyo y Karla Jiménez del Laboratorio de Ecología Acuática de la Universidad de San Francisco en Quito, Gunther Reck de ECOLAP, Andrés Vallejo de la Corporación de Salud Ambiental de Quito, Ximena Bastidas y Esteban Terneus de Fundación AGUA, Juan José Vásconez, FONAG, EMAAPQ, The Nature Conservancy y al programa Education for Nature Programme de WWF que proporciona la beca a Blanca Ríos, Clorinda Vergara y Eduardo Oyague del Museo de Entomología de la Universidad Nacional Agraria La Molina, Lima, Antoni Munné, Núria Bonada, Carolina Solà y Mireia Vila quienes junto con Narcís Prat y María Rieradevall fueron los creadores del Protocolo Ecostrimed.

Los compañeros del grupo Guadalmed de cuyo protocolo final se han tomado varios de los textos del CERA convenientemente modificados. 
Anexo 1. Formaciones vegetales frecuentes en los ecosistemas de páramo y puna en los Andes. Frequent vegetation formations in the paramo (moor) and puna ecosystems in the Andes.

\section{Ecosistema de Páramo}

Formaciones:

Páramo de pajonal de gramíneas

Páramo de Frailejones

Páramo de Chuscales

Páramo de almohadillas

Bosques achaparrados

Arbustivas

Ecosistema de Puna

Formaciones:

Puna de pajonal de gramíneas

Puna de hierbas en roseta

Puna de tolares

Puna de almohadillas

Puna de Bofedales

Bosques achaparrados

\section{Especies dominantes}

Calamagrostis, Festuca, Agrostis, Bromus, Poa, Stipa, Sysirinchium

Espeletia

Chusquea

Azorella, Werneria, Plantago

Polylepis, Buddleja, Escallonia, Hesperomeles

Miconia, Oreocallis, Weinmannia

\section{Especies dominantes}

Calamagrostis, Deyeuxia, Festuca, Agrostis, Eragrostis, Poa, Stipa, Muhlenbergia, Aristida, Eragrostis

Arenaria,Gamochaeta,Perezia,

Baccharis, Parastrephia

Azorella, Pycnophyllum

Distichia muscoides

Polylepis, Buddleja, Escallonia, Gynoxis, Puya 
Anexo 2. Estaciones de Muestreo en las cuencas de los ríos Guayllabamba (Ecuador) y Cañete (Perú). Sampling stations in the Guayllabamba (Ecuador) and Cañete (Peru) basins.

\begin{tabular}{|c|c|c|c|c|c|c|c|c|}
\hline Código & Cuenca & $\mathbf{X}$ & $\mathbf{Y}$ & Altitud & Punt. Ref & IHF & QBR-And & ABI \\
\hline SP1 & San Pedro & 786613 & 9976012 & 2305 & 74 & 50 & 5 & 24 \\
\hline SP2 & San Pedro & 784257 & 9970450 & 2386 & 70 & 61 & 20 & 3 \\
\hline SP3 & San Pedro & 783676 & 9966080 & 2450 & 64 & 45 & 0 & 18 \\
\hline SP4 & San Pedro & 783676 & 9966080 & 2460 & 70 & 63 & 20 & 3 \\
\hline SP5 & San Pedro & 776591 & 9957292 & 2631 & 72 & 70 & 25 & 21 \\
\hline SP6 & San Pedro & 773441 & 9946768 & 2825 & 76 & 54 & 25 & 16 \\
\hline SP7 & San Pedro & 772868 & 9941394 & 2935 & 82 & 63 & 30 & 25 \\
\hline M1 & Machángara & 769156 & 9964517 & 3190 & 92 & 58 & 25 & 60 \\
\hline M2 & Machángara & 775779 & 9972088 & 2799 & 72 & 56 & 20 & 11 \\
\hline M3 & Machángara & 782067 & 9978491 & 2589 & 84 & 54 & 20 & 14 \\
\hline M4(SP9) & Machángara & 788424 & 9981748 & 2142 & 82 & 52 & 20 & 15 \\
\hline M5(SP8) & Machángara & 792512 & 9992904 & 1932 & 76 & 57 & 25 & 19 \\
\hline M7 & Machángara & 785158 & 9999042 & 2382 & 78 & 57 & 25 & 19 \\
\hline M8(SP10) & Machángara & 779714 & 9959454 & 2534 & 72 & 56 & 20 & 49 \\
\hline $1.1 \mathrm{SP}$ & San Pedro & 759230 & 9935360 & 3621 & 120 & 85 & 90 & 115 \\
\hline $1.2 \mathrm{SP}$ & San Pedro & 759190 & 9935399 & 3618 & 120 & 87 & 100 & 138 \\
\hline $1.3 \mathrm{SP}$ & San Pedro & 758722 & 9932970 & 3595 & 116 & 79 & 90 & 113 \\
\hline $1.4 \mathrm{SP}$ & San Pedro & 759001 & 9932279 & 3610 & 110 & 77 & 85 & 84 \\
\hline $2.1 \mathrm{SP}$ & San Pedro & 770123 & 9937412 & 3192 & 106 & 90 & 80 & 106 \\
\hline $2.3 \mathrm{SP}$ & San Pedro & 782727 & 9952967 & 3199 & 110 & 90 & 80 & 137 \\
\hline $2.4 \mathrm{SP}$ & San Pedro & 784921 & 9950085 & 3333 & 110 & 90 & 85 & 123 \\
\hline $2.5 \mathrm{SP}$ & San Pedro & 784914 & 9950044 & 3300 & 110 & 85 & 85 & 132 \\
\hline $2.6 \mathrm{SP}$ & San Pedro & 783669 & 9951707 & 3300 & 110 & 85 & 80 & 123 \\
\hline $3.1 \mathrm{SP}$ & San Pedro & 776437 & 9953556 & 2787 & 120 & 90 & 90 & 134 \\
\hline $3.2 \mathrm{SP}$ & San Pedro & 775665 & 9953910 & 2750 & 118 & 90 & 80 & 93 \\
\hline $3.3 \mathrm{SP}$ & San Pedro & 785034 & 9954865 & 2800 & 110 & 85 & 80 & 121 \\
\hline $3.4 \mathrm{SP}$ & San Pedro & 783475 & 9954347 & 2953 & 110 & 85 & 65 & 68 \\
\hline TAMB1 & San Pedro & 772449 & 9955190 & 2812 & 102 & 78 & 60 & 124 \\
\hline TAMB2 & San Pedro & & & 2812 & 68 & 37 & 0 & 26 \\
\hline JAM1 & San Pedro & 7679 & 9937000 & 3002 & 102 & 75 & 70 & 96 \\
\hline PIl & Pita & 787558 & 9963698 & 2600 & 84 & 66 & 20 & 68 \\
\hline 3.1 PI (PI 2) & Pita & 791466 & 9956138 & 2788 & 110 & 89 & 65 & 124 \\
\hline $3.2 \mathrm{PI}$ (PI 3) & Pita & 791466 & 9956137 & 2789 & 110 & 89 & 60 & 99 \\
\hline 3.4 PI (PI 4) & Pita & 788781 & 9954114 & 2843 & 102 & 87 & 85 & 96 \\
\hline $1.1 \mathrm{PI}$ & Pita & 789230 & 9933108 & 3824 & 118 & 76 & 25 & 114 \\
\hline $1.2 \mathrm{PI}$ & Pita & 788627 & 9935085 & 3743 & 118 & 66 & 25 & 132 \\
\hline $1.3 \mathrm{PI}$ & Pita & 783876 & 9934858 & 3754 & 118 & 70 & 25 & 118 \\
\hline $1.4 \mathrm{PI}$ & Pita & 784972 & 9937998 & 3694 & 116 & 66 & 25 & 92 \\
\hline $2.1 \mathrm{PI}$ & Pita & 785620 & 9948161 & 3300 & 110 & 89 & 80 & 97 \\
\hline $2.2 \mathrm{PI}$ & Pita & 789151 & 9949452 & 3180 & 106 & 84 & 65 & 68 \\
\hline $2.3 \mathrm{PI}$ & Pita & 785420 & 9946610 & 3295 & 110 & 77 & 70 & 140 \\
\hline $2.4 \mathrm{PI}$ & Pita & 785782 & 9946620 & 3290 & 110 & 87 & 60 & 123 \\
\hline $3.3 \mathrm{PI}$ & Pita & 789094 & 9953749 & 2900 & 106 & 83 & 70 & 128 \\
\hline Sta. Clara 1 & Pita & 786712 & 9961756 & 2557 & 82 & 70 & 30 & 37 \\
\hline Sta. Clara 2 & Pita & 784916 & 9963872 & 2509 & 76 & 62 & 15 & 15 \\
\hline CA-01 & Río Cañete(Cab.) & 389558 & 8656452 & 4396 & 107 & 46 & 86,45 & 69 \\
\hline CA- 02 & Río Cañete(Cab.) & 389889 & 8656121 & 4425 & 107 & 43 & 86,45 & 92 \\
\hline $\mathrm{CA}-03$ & Río Cañete(Cab.) & 390012 & 8658592 & 4352 & 107 & 41 & 100 & 82 \\
\hline CA-04 & Río Cañete(Cab.) & 389848 & 8659023 & 4309 & 107 & 46 & 86,45 & 86 \\
\hline CA- 05 & Río Cañete(Cab.) & 395627 & 8663778 & 4276 & 113 & 36 & 99,75 & 85 \\
\hline CA-06 & Río Cañete(Cab.) & 406031 & 8663202 & 3913 & 113 & 41 & 99,75 & 66 \\
\hline CA-07 & Río Cañete(Cab.) & 407327 & 8662811 & 3935 & 107 & 41 & 73,15 & 75 \\
\hline CA- 08 & Río Cañete(Cab.) & 411685 & 8662890 & 4065 & 107 & 45 & 93,1 & 91 \\
\hline CA-09 & Río Cañete(Cab.) & 411743 & 8662876 & 4065 & 107 & 47 & 86,45 & 75 \\
\hline CA-10 & Río Cañete(Cab.) & 411084 & 8660443 & 3906 & 107 & 36 & 86,45 & 92 \\
\hline CA-11 & Río Cañete(Cab.) & 413278 & 8655824 & 3912 & 107 & 31 & 86,45 & 92 \\
\hline CA-12 & Río Cañete & 413292 & 8651242 & 3537 & 104 & 31 & 60 & 75 \\
\hline CA-13 & Río Cañete & 412549 & 8646752 & 3450 & 102 & 29 & 55 & 72 \\
\hline CA-14 & Río Cañete & 411491 & 8644779 & 3400 & 110 & 26 & 70 & 76 \\
\hline CA-15 & Miraflores & 404357 & 8643299 & 4117 & 113 & 52 & 85 & 58 \\
\hline CA-16 & Miraflores & 404562 & 8643612 & 4061 & 113 & 52 & 85 & 66 \\
\hline CA-17 & Miraflores & 411166 & 8642433 & 3300 & 112 & 65 & 95 & 66 \\
\hline CA-18 & Alis & 419486 & 8653899 & 3900 & 105 & 49 & 99,75 & 70 \\
\hline CA-19 & Alis & 419683 & 8653796 & 3900 & 105 & 42 & 86,45 & 86 \\
\hline CA-20 & Alis & 419466 & 8653506 & 3850 & 105 & 51 & 99,75 & 75 \\
\hline CA-21 & Alis & 419776 & 8649066 & 3650 & 111 & 48 & 86,45 & 74 \\
\hline CA-22 & Laraos & 417018 & 8633332 & 3736 & 108 & 33 & 55 & 63 \\
\hline CA-23 & Laraos & 415667 & 8634651 & 3600 & 104 & 37 & 75 & 82 \\
\hline CA-24 & Laraos & 412118 & 8635845 & 3050 & 110 & 68 & 85 & 119 \\
\hline CA-25 & Río Cañete & 408850 & 8632227 & 2800 & 114 & 38 & 60 & 46 \\
\hline CA-26 & Huantán & 413330 & 8622529 & 3726 & 108 & 47 & 65 & 78 \\
\hline CA-27 & Huantán & 413515 & 8622453 & 3350 & 104 & 52 & 75 & 110 \\
\hline CA-28 & Huantán & 411069 & 8624868 & 3140 & 104 & 55 & 55 & 97 \\
\hline CA-29 & Huantán & 409507 & 8625131 & 3126 & 100 & 45 & 40 & 65 \\
\hline CA-30 & Tupe & 412709 & 8591200 & 2800 & 108 & 79 & 75 & 119 \\
\hline CA-31 & Tupe & 410978 & 8591010 & 2580 & 112 & 47 & 70 & 76 \\
\hline CA-32 & Lincha & 428006 & 8585100 & 3497 & 104 & 78 & 65 & 98 \\
\hline CA-33 & Lincha & 424512 & 8583658 & 3208 & 104 & 76 & 65 & 103 \\
\hline CA-34 & Lincha & 418943 & 8581722 & 2553 & 104 & 74 & 65 & 139 \\
\hline CA-35 & Lincha & 418606 & 8580849 & 2563 & 108 & 87 & 65 & 137 \\
\hline CA-36 & Yauyos & 399700 & 8623088 & 2946 & 90 & 67 & 15 & 61 \\
\hline CA-37 & Yauyos & 400726 & 8622764 & 2881 & 90 & 49 & 40 & 35 \\
\hline CA-38 & Laraos & 413340 & 8636285 & 3223 & 96 & 49 & 15 & 78 \\
\hline CA-39 & Alis & 419173 & 8647580 & 3542 & 84 & 38 & 0 & 0 \\
\hline CA-40 & Alis & & & 3420 & 90 & 42 & 60 & 0 \\
\hline CA-41 & Alis & 414590 & 8642064 & 3218 & 90 & 51 & 10 & 45 \\
\hline CA-42 & Miraflores & 407970 & 8643198 & 3642 & 94 & 58 & 45 & 52 \\
\hline
\end{tabular}


Anexo 3. Protocolo de condiciones de referencia en ríos andinos. Reference conditions protocol in Andean Rivers.

\section{CONDICIONES DE REFERENCIA EN RIOS ANDINOS \\ CER/3 \\ Calidad Ecolögia de Rícs Andinos}

Apartado

Poco Medio Mucho

CUENCA

1.1 Cobertura de especies introducidas (Eucaliptos y Pinos especialmente)

1.2 Porcentaje de cobertura en pastos artificiales

1.3 Porcentaje de cobertura en usos urbanos

1.4 Ausencia de vegetación autóctona

1.5 Explotaciones mineras

1.6 Explotaciones ganaderas intensivas (intensivas)

$\begin{array}{lll}5 & 3 & 1 \\ 5 & 3 & 1 \\ 5 & 3 & 1 \\ 5 & 3 & 1 \\ 5 & 3 & 1 \\ 5 & 3 & 1\end{array}$

\section{HIDROLOGÍA}

2.1 Presencia de grandes presas aguas arriba del lugar

2.2 Derivaciones de agua para hidroeléctricas azudes $<10 \mathrm{~m}$

2.3 Trasvases a otras cuencas o desde otras cuencas

2.4 Derivaciones para usos en agricultura y ganadería

2.5 Derivaciones para uso en mineria

2.6 Derivaciones para uso urbano (usos domésticos e industriales)

$\begin{array}{lll}5 & 3 & 1 \\ 5 & 3 & 1 \\ 5 & 3 & 1 \\ 5 & 3 & 1 \\ 5 & 3 & 1 \\ 5 & 3 & 1\end{array}$

\section{TRAMO (Incluye ribera y zona inundación)}

3.1 Canalización del río por infraestructuras rígidas (escolleras, etc...)

3.2 Canalización del río por terraplenes

3.3 Presencia de cultivos i/ovacas y pasto en la llanura de inundación

3.4 Infraestructuras laterales (carreteras, construcciones...)

3.5 Falta de cubierta de la zona de ribera (árboles o arbustos)

$3.6 \%$ Cubierta vegetal por especies introducidas (árboles o arbustos)

$\begin{array}{lll}5 & 3 & 1 \\ 5 & 3 & 1 \\ 5 & 3 & 1 \\ 5 & 3 & 1 \\ 5 & 3 & 1 \\ 5 & 3 & 1\end{array}$

\section{LECHO}

4.1 Sustrato del lecho totalmente artificial (p.e. cemento, escollera....)

4.2 Infraestructuras transversales (p.e. azudes, vados)

4.3 Presencia de efluentes directos al río

4.4 Contaminación orgánica evidente

4.5 Contaminació minera evidente

4.6 Presencia de basuras y escombros (sea en la ribera o en el mismo lecho)

$\begin{array}{lll}5 & 3 & 1 \\ 5 & 3 & 1 \\ 5 & 3 & 1 \\ 5 & 3 & 1 \\ 5 & 3 & 1 \\ 5 & 3 & 1\end{array}$

El valor máximo del índice es de 120, el mínimo de 24.

Se considera que valores superiores a 100 son necesarios para poder considerar un punto como de referencia.

De todas formas un punto de referencia debe obtener como mínimo 20 puntos en cada apartado. 


\section{Especificaciones de cada Apartado}

El significado de Poco, Medio o Mucho en cada caso se explica a continuación. CUENCA

$1.1 \quad$ Poco $<10 \%$, Medio $10-30 \%$, Mucho $>30 \%$

$1.2 \quad$ Idem

1.3 Poco $<1 \%$, Medio $1-10 \%$, Mucho $>10 \%$. Incluye la presencia de floriculturas.

$1.4 \quad$ Poco $<10 \%$, Medio $10-50 \%$, Mucho $>50 \%$

1.5 Poco:inexistente o de muy baja intensidad, Medio:1 grande o varias de poca intensidad, Mucho: 2 grandes o muchas de pequeña intensidad

1.6 Poco: inexistente o mínimas, Medio: presencia de una gran explotación, Mucho: varias explotaciones grandes

\section{HIDROLOGIA}

$2.1 \quad$ Grandes presas $(>10 \mathrm{~m})$. Poco: inexistente, Medio: 1 , Mucho $>1$

2.2 Reducción caudal. Poco $<10 \%$, Medio $10-50 \%$, Mucho $>50 \%$

2.3 Reducción caudal. Poco: $\sin$ trasvase, Medio $<25 \%$, Mucho $>25 \%$. Incluir también trasvases de otras cuencas

2.4 Reducción caudal. Poco: sin desvío, Medio $<25 \%$, Mucho $>25 \%$ (Atención si hay múltiples pequeñas pasar de medio a mucho)

2.5 Reducción caudal. Poco: sin desvío, Medio $<25 \%$, Mucho $>25 \%$ (Atención si hay múltiples pequeñas pasar de medio a mucho)

2.6 Reducción caudal. Poco: sin desvío, Medio $<25 \%$, Mucho $>25 \%$ (Atención si hay múltiples pequeñas pasar de medio a mucho)

\section{TRAMO}

3.1 Poco: sin canalización, Medio $<25 \%$, Mucho $>25 \%$

3.2 Poco: sin canalización, Medio $<50 \%$, Mucho $>50 \%$

3.3 Poco: sin cultivos, Med io $<50 \%$, Mucho $>50 \%$

3.4 Poco: no hay, Medio: en uno de los lados, Mucho: en los dos lados (cubriendo $>10 \%$ superficie)

3.5 Poco: totalmente cubierto por vegetación nativa, Medio $>50 \%$, Mucho $<50 \%$

$$
\text { Poco: sin especies introducidas, Medio }<50 \% \text {, Mucho }>50 \%
$$

\section{LECHO}

olor

4.5 Poco: no hay minas, Medio: río con sedimentos en suspensión, Mucho: sedimentos muy abundantes y conocimiento de $\mathrm{pH}$ muy ácido o muy básico

4.6 Poco: no hay o solo aisladamente, Medio: acumulaciones d e forma aislada, Mucho: vertedero .

\section{Apartados Restrictivos}

Apartados que pueden constituir por ellos mismos una restricción para declarar un punto como de referencia por su afectación grave (puntación 1 en el apartado):

Bloque 1 1.3, 1.5

Bloque 2 2.1, 2.3. (Respecto a los apartados 2.2, 2.4, 2.5, 2.6, solo se aplica si la derivación es próxima, pero no si se ha producido ya el retorno al río del agua derivada en puntos aguas debajo dela cuenca)

Bloque 3 3.1.

Bloque 4 4.1, 4.4 y 4.5 si el valor es 1. 
Anexo 4. Índice de hábitat fluvial (IHF) (Adaptado de Pardo et al., 2002). River habitat index(IHF)(Adapted from Pardo et al., 2002)

Bloques

Puntuación

1. Inclusión rápidos

Rápidos Piedras, cantos y gravas no fijadas por sedimentos finos. Inclusión $0-30 \%$.

Piedras, cantos y gravas poco fijadas por sedimentos finos. Inclusión $30-60 \%$.

Piedras, cantos y gravas medianamente fijadas por sedimentos finos. Inclusión $>60 \%$.

TOTAL (una categoría)

\section{Frecuencia de rápidos}
Alta frecuencia de rápidos. Relación distancia entre rápidos / anchura del río $<7$
Escasa frecuencia de rápidos. Relación distancia entre rápidos / anchura del río 7 - 15
Ocurrencia ocasional de rápidos. Relación distancia entre rápidos / anchura del río 15 - 25
Constancia de flujo laminar o rápidos someros. Relación distancia entre rápidos/anchura del río $>25 \quad 4$
Sólo pozas

TOTAL (una categoria)

3. Composición del substrato (en caso de ausencia absoluta el valor debe ser 0 para cada apartado)

\begin{tabular}{|l|l|l|l|}
\hline & $\%$ Bloques y piedras & $1-10 \%$ & 2 \\
\hline & $>10 \%$ & 5 \\
\hline \multirow{2}{*}{$\%$ Cantos y gravas } & $1-10 \%$ & 2 \\
\hline & $>10 \%$ & 5 \\
\hline \multirow{2}{*}{$\%$ Arena } & $1-10 \%$ & 2 & \\
\hline & $>10 \%$ & 5 \\
\hline \multirow{2}{*}{$\%$ Limo y arcilla } & $1-10 \%$ & 2 \\
\hline
\end{tabular}

4. Regímenes de velocidad / profundidad

TOTAL (sumar categorías)

somero: $<0.5 \mathrm{~m} 4$ categorías. Lento-profundo, lento-somero, rápido-profundo y rápido-somero. 10

lento: $<0.3 \mathrm{~m} / \mathrm{s}$ Sólo 3 de las 4 categorías

8

Sólo 2 de las 4

6

Sólo 1 de las cuatro

TOTAL (una categoría)

5. Porcentaje de sombra en el cauce

Sombreado con ventanas

Totalmente en sombra

Grandes claros

Expuesto

\begin{tabular}{|l|l|l|}
\hline Sombreado con ventanas & 10 & \\
\hline Totalmente en sombra & 7 & \\
\hline Grandes claros & 5 & \\
\hline Expuesto & 3 & \\
\hline & TOTAL (una categoría)
\end{tabular}

6. Elementos heterogeneidad (si hay ausencia de hojarasca el valor debe ser 0 puntos)

\begin{tabular}{lll} 
Hojarasca & $>10 \%$ ó $<75 \%$ & 4 \\
Presencia de troncos y ramas & $<10 \%$ ó $>75 \%$ & 2 \\
Raíces expuestas & & 2 \\
Diques naturales & 2 \\
\hline
\end{tabular}

TOTAL (una categoría)

7. Cobertura de vegetación acuática (en caso de ausencia absoluta el valor debe ser cero para cada apartado)

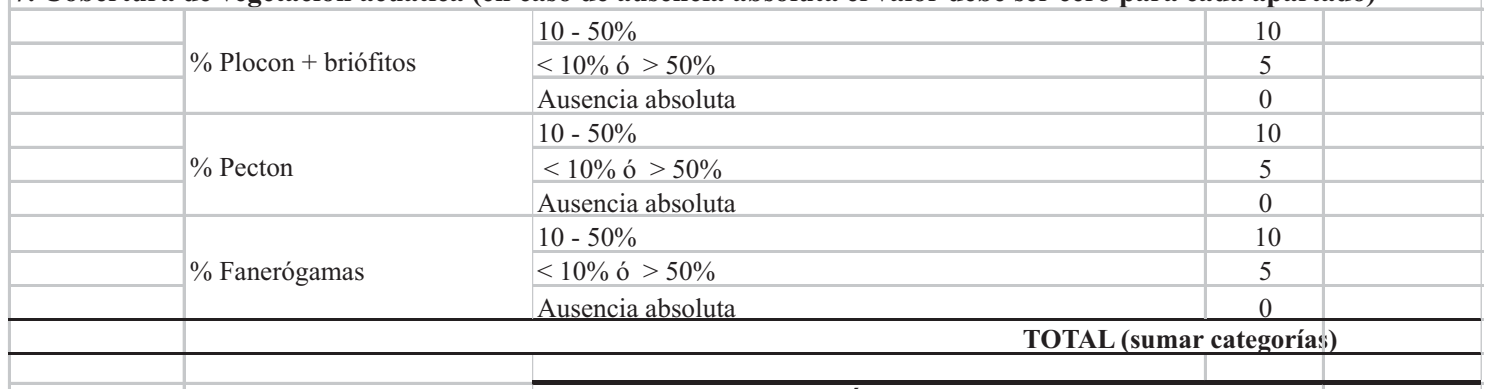


Anexo 5. Índice de calidad de la vegetación de ribera Andina (QBR-And). Andean riparian vegetation quality Index (QBR-And).

\section{ÍNDICE QBR-And \\ Calidad de la ribera para \\ Comunidades arbóreas \\ Protocolo CERA}

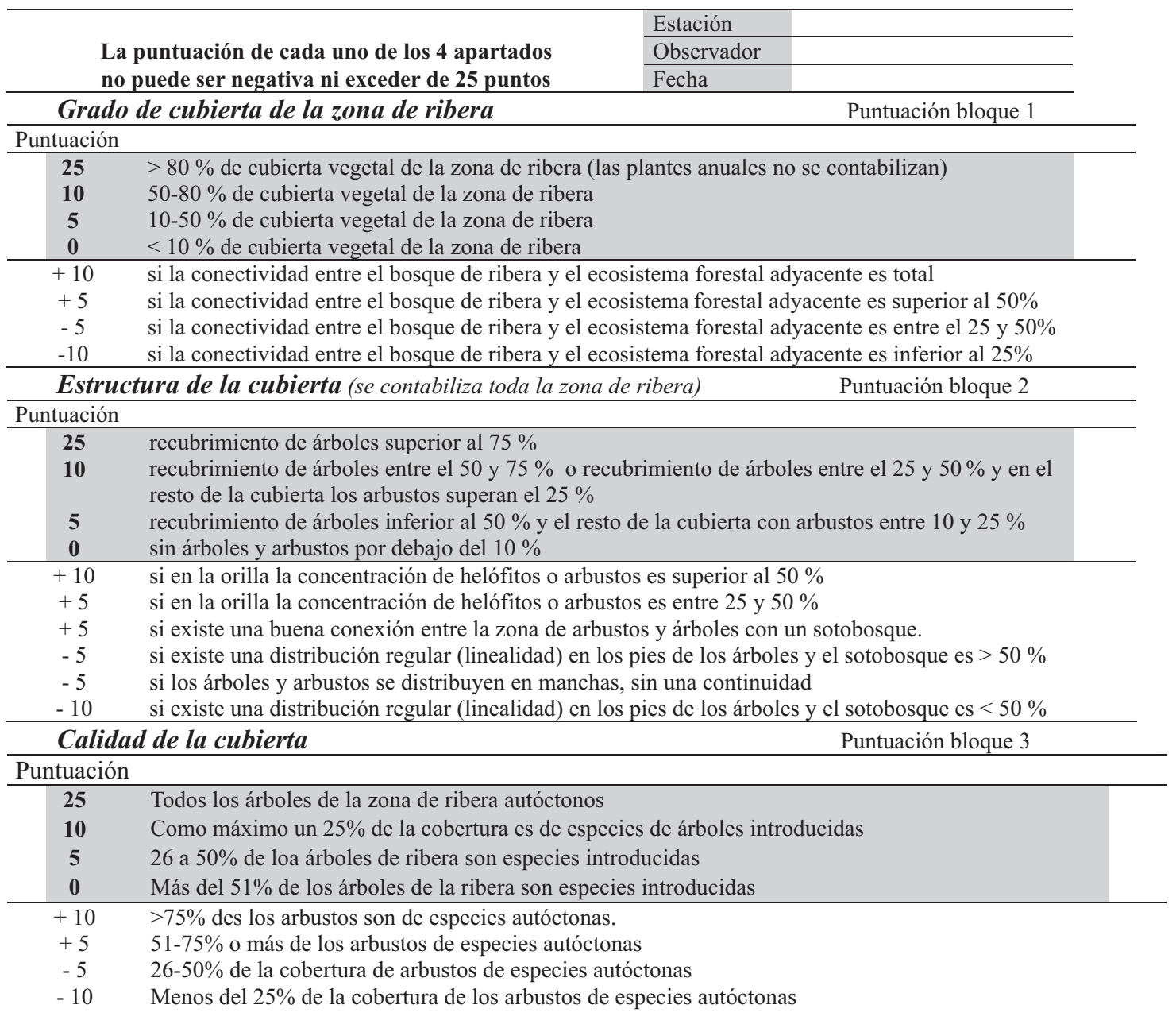

\begin{tabular}{cl}
\hline Grado de naturalidad del canal fluvial & Puntuación bloque 4 \\
\hline Puntuación & \\
\hline $\mathbf{2 5}$ & el canal del río no ha estado modificado \\
$\mathbf{1 0}$ & modificaciones de las terrazas adyacentes al lecho del río con reducción del canal \\
$\mathbf{5}$ & signos de alteración y estructuras rígidas intermitentes que modifican el canal del río \\
$\mathbf{0}$ & rí canalizado en la totalidad del tramo \\
\hline-10 & si existe alguna estructura sólida dentro del lecho del río \\
-10 & si existe alguna presa o otra infraestructura transversal en el lecho del río \\
-5 & si hay basuras en el tramo de muestreo de forma puntual pero abundantes \\
\hline Puntuación final (suma de las anteriores puntuaciones)
\end{tabular}

Puntuación final (suma de las anteriores puntuaciones) 


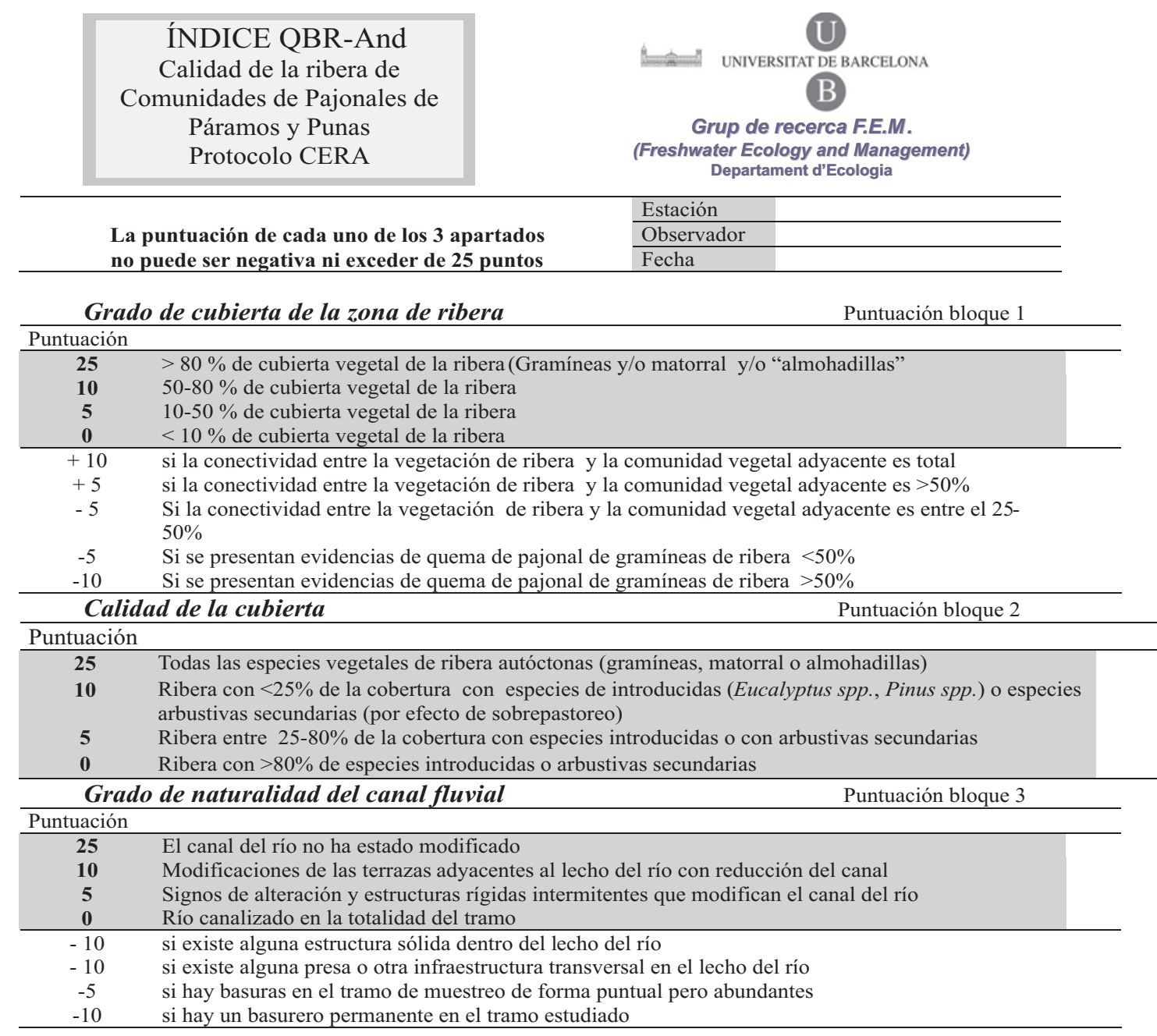

Puntuación final (suma de las anteriores puntuaciones) 
Anexo 6. Especies vegetales nativas e introducidas en los Andes. Native and introduced vegetation species in the Andes.

\begin{tabular}{|c|c|}
\hline \multicolumn{2}{|l|}{ Especies nativas } \\
\hline Familia & Especie \\
\hline \multicolumn{2}{|r|}{ Árboles } \\
\hline Actinidaceae & Sauraria spp \\
\hline Anacardiaceae & Schinus spp \\
\hline Apocynaceae & Aspidosperma quebracho-blanco \\
\hline Araliaceae & Oreopanax spp \\
\hline Asteraceae & Barnadesia spinosa \\
\hline Asteraceae & Gynoxis spp \\
\hline Berberidaceae & Berberis spp \\
\hline Betulaceae & Alnus acuminata \\
\hline Buddlejaceae & Buddleja spp \\
\hline Caesalpinaceae & Senna weddelliana \\
\hline Clethraceae & Clethra spp \\
\hline Clusiaceae & Clusia spp \\
\hline Cunoniaceae & Weinmannia spp \\
\hline Eaelocarpaceae & Vallea stipulans \\
\hline Eaelocarpaceae & Vallea spp \\
\hline Fabaceae & Cercidium andicola \\
\hline Fabaceae & Acacia feddeana \\
\hline Mimosaceae & Prosopis spp \\
\hline Poaceae & Cortaderia nitida \\
\hline Podocarpaceae & Podocarpus spp \\
\hline Podocarpaceae & Prumnopitys spp \\
\hline Rosaceae & Polylepis spp \\
\hline Rosaceae & Hesperomeles spp \\
\hline Rutaceae & Schinopsis haenkeana \\
\hline Saxifragaceae & Escallonia spp \\
\hline Solanaceae & Solanum culcullatum \\
\hline Solanaceae & Solanum felinum \\
\hline Verbenaceae & Citharexylum spp \\
\hline Zygophyllaceae & Bulnesia rivas-martinezii \\
\hline & Arbustos \\
\hline Asteraceae & Parastrephia spp \\
\hline Asteraceae & Baccharis spp \\
\hline Asteraceae & Diplostephium spp \\
\hline Grossulariaceae & Ribes spp \\
\hline Melastomataceae & Brachyotum spp \\
\hline Melastomataceae & Miconia spp \\
\hline \multicolumn{2}{|c|}{ Hierbas y arbustos sumergidos } \\
\hline Apiaceae & Aracha sculenta \\
\hline Brassicaceae & Nasturtium spp \\
\hline Gesneriaceae & Koehleria spp \\
\hline Gesneriaceae & Besteria spp \\
\hline Gunneraceae & Gunnera spp \\
\hline
\end{tabular}

\begin{tabular}{ll}
\hline Especies introducidas & \\
\hline Familia & Especie \\
\hline & Árboles \\
\hline Fabaceae & Robinia pseudo-acacia \\
Myrtaceae & Eucalyptus spp \\
Pinaceae & Pinus spp \\
Platanaceae & Platanus x hispanica \\
Salicaceae & Populus deltoides \\
Salicaceae & Populus nigra spp italica \\
Salicaceae & Populus canadensis \\
Salicaceae & Salix babylonica \\
Simaroubaceae & Ailanthus altissima \\
Ulmaceae & Celtis australis \\
Frutales diversos & \\
\hline
\end{tabular}

Información recopilada por cortesía de Vlastimil Zak (Universidad San Francisco de Quito) y desde Ibisch et al. (2002) y Moraes et al. (2006). 
Anexo 7. Propuesta para el Índice Biótico Andino (ABI) para evaluar la calidad del agua de los ríos andinos. Proposal for the Andean Biotic Index ABI) to evaluate water quality in Andean Streams.

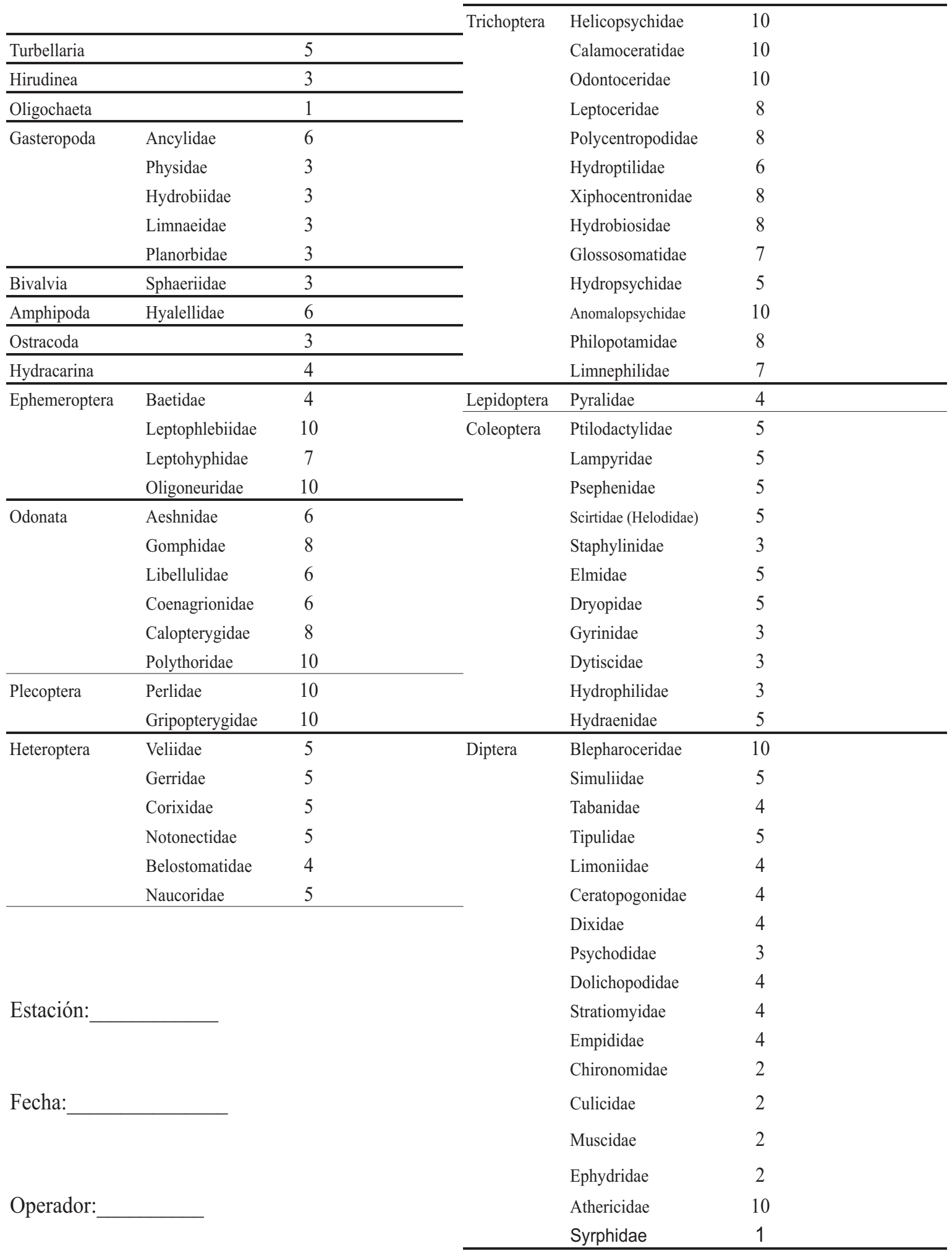




\section{BIBLIOGRAFÍA}

AGUIRRE, Z., L. P. KVIST \& O. SÁNCHEZ. 2006. Bosques secos en Ecuador y su diversidad. In: Botánica Económica de los Andes Centrales. M. Moraes, B. Øllgaard, L. P. Kvist, F. Borchsenius \& H. Balsev (eds.): 162-187. Universidad Mayor de San Andres, La Paz. Bolivia.

ALBA-TERCEDOR, J. \& A. SÁNCHEZ-ORTEGA. 1988. Un método rápido y simple para evaluar la calidad biológica de las aguas corrientes basado en el de Hellawell (1978). Limnetica, 4: 51-56.

ALBA-TERCEDOR, J., P. JÁIMEZ-CUÉLLAR, M. ÁLVAREZ, J. AVILÉS, N. BONADA, J. CASAS, A. MELLADO, I. ORTEGA, M. PARDO, N. PRAT, M. RIERADEVALL, S. ROBLES, C. SÁINZ-CANTERO, A. SÁNCHEZ-ORTEGA, M.L. SUÁREZ, M. TORO, M. R. VIDALABARCA, S. VIVAS \& C. ZAMORA-MUÑOZ. 2002. Caracterización del estado ecológico de ríos mediterráneos ibéricos mediante el índice IBMWP (antes BMWP'). Limnetica, 21(3-4): 175-182.

ALLAN, J. D. 1995. Stream Ecology: Structure and funtion of running waters. $1^{\mathrm{a}}$ ed. Chapman $\&$ Hall. $388 \mathrm{pp}$.

ARAUJO-MURAKAMI, A. \& F. S. ZENTENO. R. 2006. Bosques de los Andes Orientales de Bolivia y sus especies útiles. In: Botánica Económica de los Andes Centrales. M. Moraes, B. Øllgaard, L. P. Kvist, F. Borchsenius \& H. Balsev (eds.): 146161. Universidad Mayor de San Andres, La Paz. Bolivia.

BALLESTEROS, Y. V., M. del C. ZÚÑIGA DE CARDOZO, \& A. M. ROJAS DE HERNÁNDEZ. 1997. Distribution and Structure of the order Trichoptera in various drainages of the Cauca River basin, Colombia, and their relationship to water quality. Proceedings $8^{\circ}$ International Symposium on Trichoptera 19-23.

BEISEL, J-N., P. USSEGLIO-POLATERA, \& J. C. MORETEAU. 2000. The Spatial Heterogeneity of a river bottom: a key factor determining macroinvertebrate communities. Hydrobiologia, 422/423: 163-171

BONADA, N., N. PRAT, A. MUNNÉ, M. RIERADEVALL, J. ALBA-TERCEDOR, M. ÁLVAREZ, J. AVILÉS, J. CASAS, P. JÁIMEZ-CUÉLLAR, A. MELLADO, M. ORTEGA, I. PARDO, S. ROBLES, C. SÁINZ-CANTERO, A. SÁNCHEZORTEGA, M. L. SUÁREZ, M. TORO, M. R.
VIDAL-ABARCA, S. VIVAS \& C. ZAMORAMUÑOZ. 2002. Criterios para la selección de condiciones de referencia en los ríos mediterráneos. Resultados del proyecto GUADALMED1. Limnetica, 21(3-4): 99-114.

BOON P. J. \& D. L. HOWELL. 1997. Freshwater quality: Defining the indefinable?. Scottish Natural heritage. Edinburgh. 552 pp.

CHAPMAN, D. (Ed.). 1996. Water Quality Assessments. A guide to use of biota, sediments and water in environmental monitoring. Chapman \& Hall, Cambridge. 660 pp.

CHAVES, M. L., J. L. COSTA, P. CHAINHO, M. J. COSTA, \& N. PRAT. 2006. Selection and validation of reference sites in small river basins. Hydrobiologia, 573: 133-154.

CHURCHILL, S. P., H. BALSLEV, E. FORERO, \& J. L. LUTEYN (Eds.). 1995. Biodiversity and Conservation of Neotropical Montane Forests. New York Botanical Garden, New York. 702 pp.

CLARKE K. R. \& R. M. WARWICK. 1994. Change in marine communities: an approach to statistical analysis and interpretation. Natural Environmental Research Council. Plymouth, Reino Unido. $144 \mathrm{pp}$.

CORVALÁN, J. 1990. Geologic-Tectonic Framework of the Andean Region. In: Geology of The Andes and Its relation to Hydrocarbon and Mineral resources. G. Ericksen, M. Cañas, \& J. A. Reinemund (eds.): 1-11. Huston, Texas.

D.O.C.E. 2000. Directiva 2000/60/EC del Parlamento Europeo y del Consejo de 23 de octubre de 2000 por la que se establece un marco comunitario de actuación en el ámbito de la política de aguas. D.O.C.E. L 327 de 22.12.00. 69 pp.

FERNÁNDEZ, H. \& E. DOMÍNGUEZ. 2001. Guía para la determinación de artrópodos bentónicos sudamericanos. Universidad Nacional de Tucumán. Tucumán, Argentina. 282 pp.

FIGUEROA, R., C. VALDOVINOS, E. ARAYA \& O. PARRA. 2003. Macroinvertebrados bentónicos como indicadores de calidad de agua del sur de Chile. Revista Chilena de Historia Natural, 76: 275-285.

FLORY, E. \& A. MILNER. 1999. Influence of Riparian Vegetation on Invertebrate Assemblages in a Recently Formed Stream in Glacier Bay National Park, Alaska. J. N. Am. Benthol Soc., 18(2): 261273.

FOSSATI, O., J. WASSON, C. HÉRY, G. SALINAS, \& R. MARÍN. 2001. Impact of sediment releases 
on water chemistry and macroinvertebrate communities in clear water Andean streams (Bolivia). Arch. Hydrobiol., 151(1): 33-50.

GARCÍA, E. \& S. G. BECK. 2006. Puna. In: Botánica Económica de los Andes Centrales. M. Moraes, B. Øllgaard, L. P. Kvist, F. Borchsenius \& H. Balsev (eds.): 51-76. Universidad Mayor de San Andres, La Paz. Bolivia.

GREGORY-WODZICKI, K. 2000. Uplift history of the Central and Northern Andes: A review. GSA Bulletin, 112(7): 1091-1105.

IBISCH, P. L., N. V. ARAUJO \& A. CARRETERO (EDS). 2002. Mapa de los Bosques nativos Andinos de Bolivia. Memoria Explicativa. FANPROBONA. La Paz. Bolivia. 60 pp.

JACOBSEN, D. 1998. The Effect of Organic Pollution on the Macroinvertebrate Fauna of Ecuadorian Highland Streams. Arch. Hydrobiol., 143(2): 179195.

JACOBSEN, D., R. SCHULTZ, \& A. ENCALADA. 1997. Structure and diversity of stream invertebrate assemblages: the influence of temperature with altitude and latitude. Freshwat. Biol., 38: 247-261.

JACOBSEN, D. \& A. ENCALADA. 1998. The Macroinvertebrate fauna of Ecuadorian high-land streams in the wet and dry season. Arch. Hydrobiol., 142(1): 53-70.

JACOBSEN, D., S. ROSTGAARD, \& J. J. VÁSCONEZ. 2003. Are Macroinvertebrates in high altitute streams affected by oxygen deficiency? Freshwat. Biol., 48: 2025- 2032.

JACOBSEN, D. \& R. MARÍN. 2007. Bolivian Altiplano streams with low richness of macroinvertebrates and large diel fluctuations in temperature and dissolved oxygen. Aquatic.Ecol., 41(3): 10.1007/s10452-007-9127-x

JÁIMEZ-CUÉLLAR, P., VIVAS, S., BONADA, N., ROBLES, S., MELLADO, A., ÁlVAREZ, M., ALBA-TERCEDOR, J., AVILÉS, J., CASAS, J., ORTEGA, M., PARDO, I., PRAT, N., RIERADEVALL, M., SÁINZ-CANTERO, C., SÁNCHEZORTEGA, A., SUÁREZ, M. L., TORO, M., VIDAL-ABARCA, M. R. \& ZAMORA-MUÑOZ, C. 2002. Protocolo GUADALMED (PRECE). Limnetica, 21(3-4): 187-204.

KESSLER, M. 2006. Bosques de Polylepis. In: Botánica Económica de los Andes Centrales. M. Moraes, B. Øllgaard, L. P. Kvist, F. Borchsenius \& H. Balsev (eds.): 110-120. Universidad Mayor de San Andres, La Paz. Bolivia.
KESSLER, M. y P. DRIESCH. 1993. Causas e historia de la destrucción de bosques Altoandinos en Bolivia. Ecología en Bolivia, 21: 1-18.

MACHADO, T., A. URÁN, J. RAMÍREZ, G. LENIS, A.WILLS, N. VILLEGAS, M. BLANDÓN \& M. GONZÁLEZ. 1997. Aspecto Biológico y Fisicoquímico del río Medellín. Estado Social, Económico y Ambiental del Río Medellín. Tomo I. Instituto Mi Río Universidad de Antioquia. Medellín, Colombia. 184 pp.

MENA, P. \& R. HOFSTEDE. 2006. Los Páramos Ecuatorianos. In: Botánica Económica de los Andes Centrales. M. Moraes, B. Øllgaard, L. P. Kvist, F. Borchsenius \& H. Balsev (eds.): 91-109. Universidad Mayor de San Andres, La Paz. Bolivia.

MONAGHAN, K. A., M. P. PECK, P. A. BREWIN, M. MASIERO, E. ZARATE, P. TURCOTTE, \& S. J. ORMEROD. 2000. Macroinvertebrate distribution in Ecuadorian hill streams: the effects of altitude and land use. Arch. Hydrobiol., 149(3): 421440.

MUNNÉ, A., C. SOLÀ \& N. PRAT. 1998a. QBR: Un índice rápido para la evaluación de la calidad de los ecosistemas de ribera. Tecnología del Agua, 175: 20-37.

MUNNÉ, A., C. SOLÀ, M. RIERADEVALL \& N. PRAT. 1998b. Índex QBR. Mètode per a l'avaluació de la qualitat dels ecosistemes de ribera. Estudis de la Qualitat Ecològica dels Rius (4). Diputació de Barcelona. Àrea de Medi Ambient. $28 \mathrm{pp}$.

MUNNÉ, A., N. PRAT, C. SOLÀ, N. BONADA, \& M. RIERADEVALL. 2003. A simple field method for assessing the ecological quality of riparian habitat in rivers and streams. QBR index. Aquatic Conserv.: Mar. Freshw. Ecosyst., 13: 147-164.

MUNNÉ, A. \& N. PRAT. 2008. Use of macroinvertebrate-based multimetric indices for quality evaluation in Spanish Mediterranean rivers. An intercalibration approach with the IBMWP index. Hydrobiologia (en prensa).

MYERS, N., R. A. MITTERMEIER, C. G. MITTERMEIER, G. A. B. DA FONSECA, \& J. KENT. 2000. Biodiversity hotspots for conservation priorities. Nature, 403: 853-858.

PALMER, M. A. \& N. L. POFF. 1997. The Influence of Environmental Heterogeneity on patterns and Processes in Streams. J. N. Am. Benthol. Soc., 16(1): 169-173.

PARDO, I., M. ÁlVAREZ, J. L. MORENO, S. VIVAS, N. BONADA, J. ALBA-TERECEDOR, P. 
JAIMEZ-CUELLAR, G. MOYA, N. PRAT, N. ROBLES, M. TORO, \& M. R. VIDAL-ABARCA. 2002. El hábitat de los ríos mediterráneos. Diseño de un índice de diversidad de hábitat. Limnetica, 21(3-4): 115-134.

PARRA, O. 1992. Escenario del sistema cuenca del río Biobío y aporte del proyecto EULA a su desarrollo sustentable. In: Del suelo y manejo de los recursos hídricos en la cuenca del río Biobío. F. Faranda \& O. Parra (eds): Vol 2: 91-103. Monografías EULA-Chile: Serie Actas de Seminarios Científicos.

POSADA, A., G. ROLDÁN \& J. J. RAMÍREZ. 2000. Caracterización fisicoquímica y biológica de la calidad de aguas de la cuenca de la quebrada Piedras Blancas, Antioquia, Colombia. Revista de Biología Tropical, 48(1): 59-70.

PRAT, N., A. MUNNÉ, M. RIERADEVALL, C. SOLÀ \& N. BONADA. 2000. ECOSTRIMED. Protocol per determinar l'estat ecològic dels rius mediterranis. Diputació de Barcelona. Àrea de Medi Ambient (Estudis de la Qualitat Ecològica dels Rius, 8). 94 pp.

PRINGLE, C. M., R. J. NAIMAN, G. BRETSCHKO, J. R. KARR, M. W. OSWOOD, J. R. WEBSTER, R. L. WELCOMME \& M. J. WINTERBOURN. 1988. Patch dynamics in lotic streams: The stream as a mosaic. J. N. Am. Benthol. Soc., 7: 503-524.

PRINGLE, C. M., F. N. SCATENA, P. PAABYHANSEN \& M. NÚÑEZ FERRERA. 2000. River conservation in Latin America and the Caribbean. In: Global Perspectives on River Conservation. Science, Policy and Practice. P. J. Boon, B. R Davies \& G. E. Petts (eds.): 41-77 John Wiley and Sons Ltd.

RAMÍREZ, A., P. PAABY, C. M. PRINGLE \& G. AGÜERO. 1998. Effect of habitat type on benthic macroinvertebrates in two lowland tropical streams, Costa Rica. Rev. Biol. Trop.. Supl., 6: 201213.

RINCÓN, M. E. 1996. Aspectos Bioecológicos de los tricopteros de la quebrada Carrizal (Boyacá, Colombia). Rev. Colomb. Entomol., 22(1): 53-60.

ROLDÁN, G., J. BUILES, C. M. TRUJILLO \& A. SUÁREZ. 1973. Efectos de la contaminación industrial y doméstica sobre la fauna béntica del río Medellín. Actualidades Biológicas, 2(4):54-64. Universidad de Antioquia, Medellín, Colombia.

ROLDÁN, G. 1988. Guía para el estudio de los macroinvertebrados acuáticos del Departamento de
Antioquia. Fondo FEN Conciencias. Universidad de Antioquia. Colombia. 217 pp.

ROLDÁN, G. 1999. Los macroinvertebrados y su valor como indicadores de la calidad del agua. Revista de la Academia Colombiana de Ciencias Exactas, Físicas y Naturales, 23(88): 375-387.

SÁNCHEZ-VEGA, I. \& M. O. DILLON. 2006. Jalcas In: Botánica Económica de los Andes Centrales. M. Moraes, B. Øllgaard, L. P. Kvist, F. Borchsenius \& H. Balsev (eds.): 77-90. Universidad Mayor de San Andres, La Paz. Bolivia.

STATSOFT, INC. 1999. STATISTICA for Windows (Computer Program Manual). StatSoft, Inc, Tucsa.

SUÁREZ-ALONSO, M. L. \& M. R. VIDAL-ABARCA. 2000. Aplicación del índice de calidad del bosque de ribera, QBR (Munné et al., 1998) a los cauces fluviales de la cuenca del río Segura. Tecnología del Agua, 201: 33-45.

SUÁREZ-ALONSO, M. L., M. R. VIDAL-ABARCA, M. M. SÁNCHEZ-MONTOYA, J. ALBATERCEDOR, M. ÁLVAREZ, J. AVILÉS, N. BONADA, J. CASAS, P. JÁIMEZ-CUELLAR, A. MUNNÉ, I. PARDO, N. PRAT, M. RIERADEVALL, M. J. SALINAS, M. TORO \& S. VIVAS. 2002. La ribera de los ríos mediterráneos y su calidad: el uso del índice QBR. Limnetica, 21(34): 135-148.

TANIGUCHI, H. \& M. TOKESHI.2004. Effects of Habitat Complexity on Benthic Assemblages in a variable environment. Freshwat. Biol., 49: 11641178

UNITED NATIONS ENVIRONMENTAL PROGRAMME (UNEP). 2002. Latin America and the Caribbean. Global Environment Outlook 3 (GEO) Nairobi, Kenya. 416 pp.

VANNOTE, R. L., G. W. MINSHALL, K. W. CUMMINS, J. R. SEDELL \& C. E. CUSHING. 1980. The river continuum concept. Can. J. Fish. Aquat. Sci., 37: 130-137.

WALLIN, M., T. WIEDERHOLM \& R. K. JOHNSON. 2003. Guidance on establishing reference conditions and ecological status class boundaries for inland surface waters. Resumen reunion REFCOND. CIS Working Group 2.3. 93 pp.

WINTERBOURN, M. J. \& W. F. MCDIFFETT. 1996. Benthic faunas of streams of low $\mathrm{pH}$ but contrasting water chemistry in New Zealand. Hydrobiologia, 341(2): 101-111.

YOUNG, K. R. 2006. Bosques Húmedos. En: Botánica Económica de los Andes Centrales. M. Moraes, B. Øllgaard, L. P. Kvist, F. Borchsenius \& H. 
Balsev (eds.): 121-129. Universidad Mayor de San Andres, La Paz. Bolivia.

ZÚÑIGA DE CARDOZO, M. Del C., A. M. ROJAS De HERNÁNDEZ \& S. MOSQUERA. 1997. Biological aspects of Ephemeroptera in rivers of southwestern Colombia (South America). In: Ephemeroptera and Plecoptera: Biology-EcologySystematics. P. Landolt \& M. Sartori (eds): 261268. Mauron-Tinguely and Lachat S.A. Fribourg, Switzerland. 